\title{
statacons: An SCons-based build tool for Stata
}

\author{
Raymond P. Guiteras \\ North Carolina State University \\ Raleigh, N.C., USA \\ rpguiter@ncsu.edu \\ Brian Quistorff \\ Bureau of Economic Analysis \\ Washington, D.C., USA \\ Brian.Quistorff@bea.gov
}

\author{
Ahnjeong Kim \\ North Carolina State University \\ Raleigh, N.C., USA \\ akim7@ncsu.edu \\ Clayson Shumway \\ North Carolina State University \\ Raleigh, N.C., USA \\ cshumwa@ncsu.edu
}

\begin{abstract}
This paper presents statacons, an SCons-based build tool for Stata. Because of the integration of Stata and Python in recent versions of Stata, we are able to adapt SCons for Stata workflows without the use of an external shell or extensive configuration. We discuss the usefulness of build tools generally, provide examples of the use of statacons in Stata workflows, present key elements of the syntax of statacons, and discuss extensions, alternatives, and limitations. Appendices provide installation instructions and recommendations for collaborative workflows.
\end{abstract}

Keywords: statacons, reproducible research, build tools, scons, Python

\section{Build Tools}

Transparency and reproducibility are increasingly important norms in empirical science (Wilson et al. 2014, Stodden et al. 2018; Christensen et al. 2019; Orozco et al. 2020). Public posting of data and code, use of version control software, and literate programming are all becoming more common, both in general and with Stata users specifically.

One set of tools that have not received as much attention in Stata are build tools (Mokhov et al. 2018). Build tools track dependencies throughout a project: which inputs and programs are used to create which outputs, which outputs are subsequently used or combined to create final outputs, etc. A project with a workflow encoded in a build script can be replicated from beginning to end with one command. Just as importantly, when inputs or code change, a build tool can decide exactly which outputs need to be rebuilt, avoiding both errors of omission - failing to update outputs when inputs change - and errors of inclusion - unnecessarily re-running potentially time-consuming code when no relevant inputs have changed.

Stata users already often instinctively create a rudimentary build tool: a "master" do-file that lists all of a project's do-files in order. This is adequate for simple projects, but has limitations in complex workflows. First, a list of do-files provides no information on what produces what, and what the relationships among different inputs and outputs are 11 This makes it difficult to trace outputs back to their source or debug problems.

1. Comments in a master do-file can provide some documentation of inputs and outputs, but drift be- 
Second, a master do-file does not handle the tradeoff between errors of omission and errors of inclusion well. Running a master do-file from beginning to end should avoid errors of omission, but is inefficient if some sections of the project take a long time to run. It would not make sense to re-run a computationally intensive estimation or bootstrapping procedure just to adjust the formatting of some graphs. Again, users often intuitively handle these problems by commenting out lines of the master do-file or setting up switches to skip sections of code, and this is usually fine for simple projects.

However, as projects become more complex, an approach like a master do-file can be inadequate. For example, many projects have some or all of the following characteristics: merging multiple input datasets and producing several inter-related datasets for analysis; results of some analyses being used as inputs for others (e.g., estimation results used in subsequent simulations); multiple collaborators working on different aspects of the project simultaneously, using version control software and file-sharing platforms to share code and files; combining Stata tasks with non-Stata tasks, e.g., compiling text, tables and figures into a PDF. In such cases, it is easy to lose track of which parts of a project need to be rebuilt. Similarly, in projects with multiple collaborators (or a single researcher collaborating with her past self), one collaborator may not know about the dependence of one part of the project on another.

Software developers face similar problems, and have developed sophisticated build tools to deal with them. Two ideas are central: first, the relationships between inputs, code and outputs are encoded in a build script; second, the build software uses this script and information it collects on the state of the project to manage the build, deciding what is up to date and what needs to be built or rebuilt. The build script itself provides living documentation of the full structure of the project, and the build tool can provide the user with the status of of any particular component, or of all components.

The use of build tools does not appear to be common among Stata users. We suspect the main barriers to adoption are: first, lack of awareness of the existence and value of build tools; second, the startup cost of configuring the tool, system and Stata so that they can work together; third, the startup cost of learning how to use these tools.

In this paper and with statacons, we attempt to address all three of these barriers. First, we will show in our examples that statacons can be used to manage both simple and complex workflows efficiently. Second, because of the integration of Python and Stata in recent versions (16+), as well as our own adaptations, we are able to make SCons, a popular Python-based build tool, accessible in Stata through our command statacons. With a minimal amount of configuration, Stata users can use statacons directly in Stata and write the build scripts, SConstructs, directly in Stata's do-file editor, taking advantage of the editor's Python syntax highlighting ${ }^{2}$ Third, our examples in this paper, as well as additional examples in our companion web tutorial and project

tween comments and the true content of code is a notorious source of confusion and error Gentzkow and Shapiro 2014). Furthermore, after an initial setup, virtually all the effort in using a build tool consists in encoding inputs and outputs, so on the margin there is little additional effort involved in using a build tool relative to providing comments in a master do-file.

2. SConstruct files written for statacons are standard SConstructs and will work without modification in standard SCons, so users who prefer the terminal can continue to work there. 
Wiki, can easily be adapted to users' own work.

More specifically, statacons offers the following benefits: (a) not requiring changes to existing Stata code or the structure of existing workflows; (b) rebuilding exactly those parts of a project that need to be rebuilt at any given moment, with neither errors of inclusion nor omission; (c) complementing existing workflow practices, including literate programming and use of version control software and file-sharing platforms; (d) allowing easy extensiblity; and (e) operating fully within Stata.

Our paper proceeds as follows: in Section 2, we use a simple example of managing a Stata workflow with statacons and an SConstruct to introduce basic concepts, vocabulary and mechanics; in Section 3 , we provide the syntax of the statacons command and SConstructs; in Section 4, we present an applied example of using statacons to manage the complex workflow of an empirical project (Shumway and Wilson 2021); in Section 5. we describe some of the technical details of our adaptation of SCons to Stata; in Section 6, we discuss extensions, alternative approaches, and limitations; Section 7 concludes. We include a detailed installation guide at the end of the paper. Online Appendices discuss parallel builds, illustrate some additional advanced features, and provide examples of the use of statacons in collaborative projects using version control software and shared files. Our project web pag $\AA^{3}$ provides installation instructions and hosts a companion web tutorial based on Software Carpentry's "Automation and Make" tutorial (Jackson 2016) with several additional worked examples ${ }^{4}$ The statacons package is available at our project repository 5 where we have posted complete replication code and data for the Introductory Example and key code from the Applied Example, as well as a project Wiki with examples of additional tools.

\section{Introduction by Example}

In this section, we use a simple example to introduce the basic concepts, terminology and mechanics of managing a workflow with statacons. Replication code and data for this example are provided in stataconsIntro.zip, posted to our repository 6

Our project has two steps, using the following two two do-files:

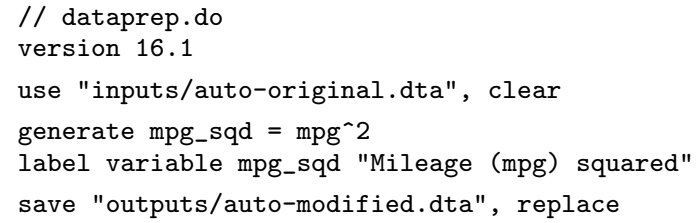

3. https://bquistorff.github.io/statacons

4. https://bquistorff.github.io/statacons/swc

5. https://github.com/bquistorff/statacons

6. https://github.com/bquistorff/statacons/raw/main/examples/stataconsIntro.zip 


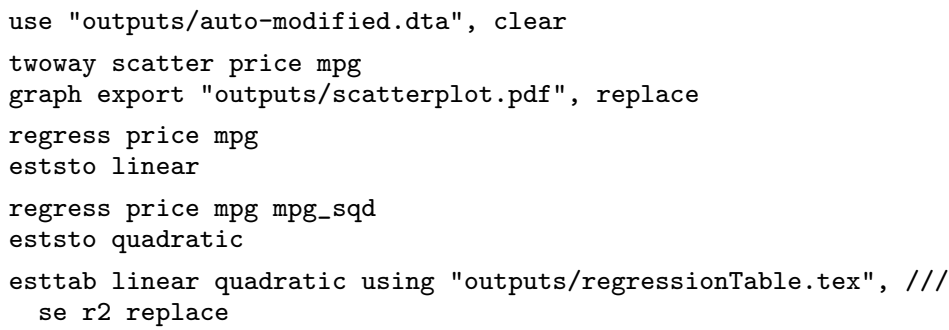

In the first step, the do-file dataprep.do takes an input file, auto-original.dta, creates a new variable mpg_sqd, which is the square of $\mathrm{mpg}$, and saves the resulting dataset as auto-modified.dta. In the second step, the do-file analysis.do uses auto-modified.dta to create a scatterplot of price against mpg, saved as scatterplot.pdf, and regresses price on mpg and mpg_sqd, exporting the regression results to regressionTable.tex.

This workflow is represented visually in Figure 1 and can easily be managed by a simple master do-file:

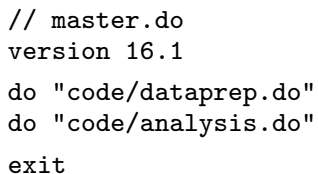

When using a build tool, it is useful to think about the workflow differently. Rather than think about a sequence of do-files in a particular order, we think about the targets that we want to build and the dependencies that we use to create these targets. In this example, the ultimate targets are scatterplot.pdf and regressionTable.tex. The dependencies for these targets are the source code analysis.do and the dataset auto-modified.dta. The dataset auto-modified.dta is itself a target, with dependencies dataprep.do and auto-original.dta.

This workflow is depicted visually in Figure 2

To use statacons, we encode these relationships in an SConstruct file:

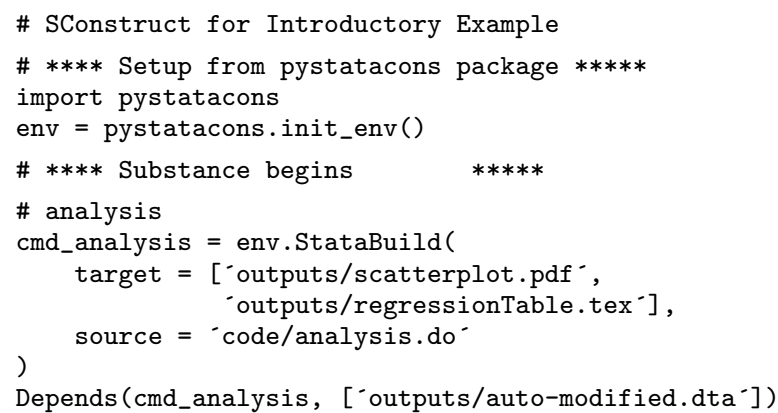




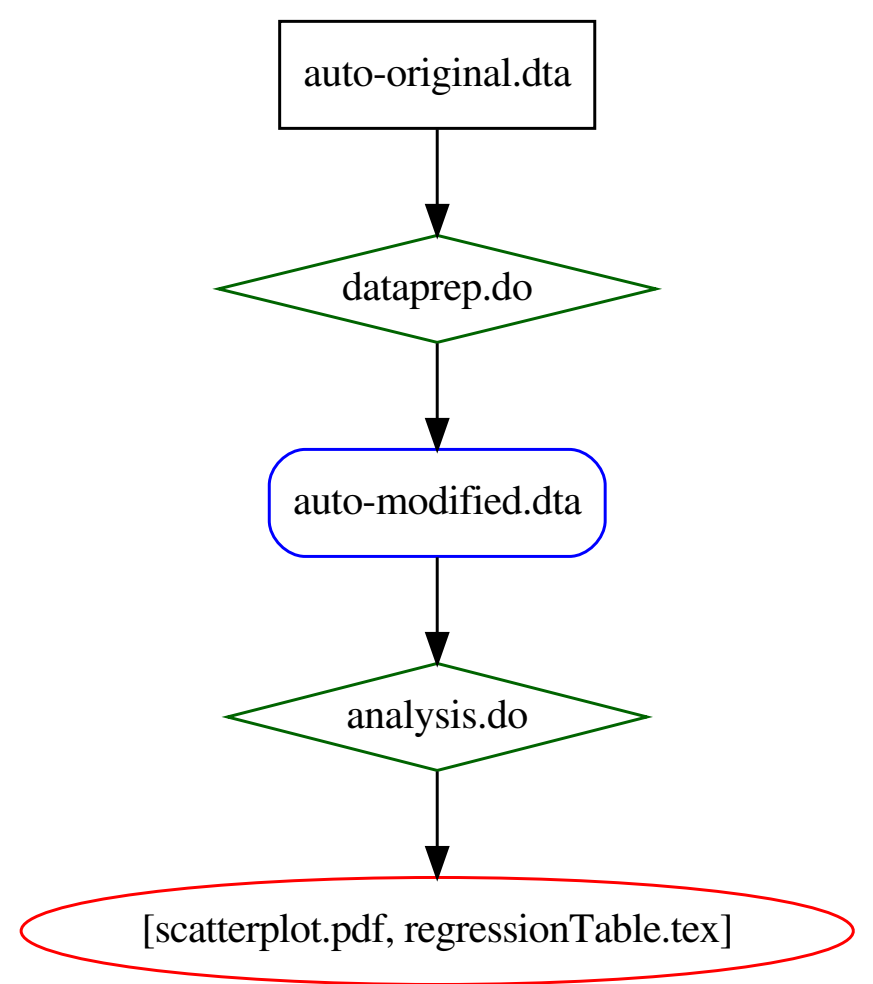

Figure 1: Linear Workflow for Introductory Example 

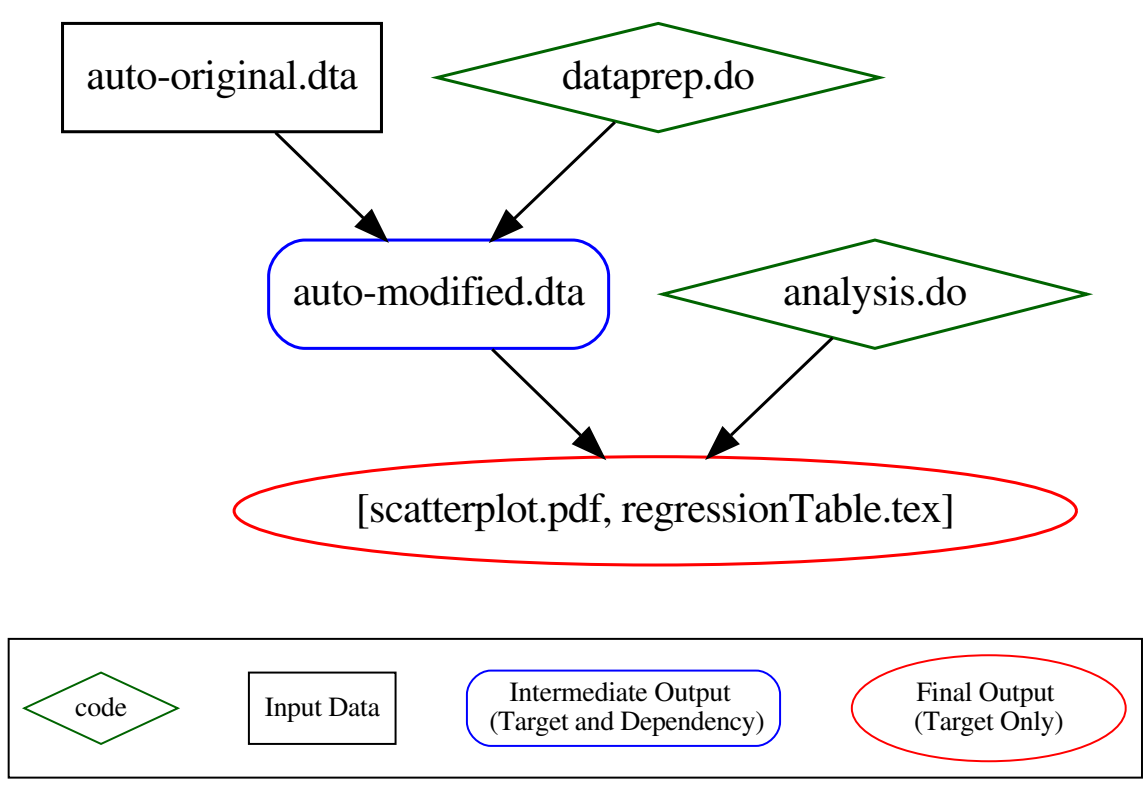

Figure 2: Target-Build Workflow for Introductory Example

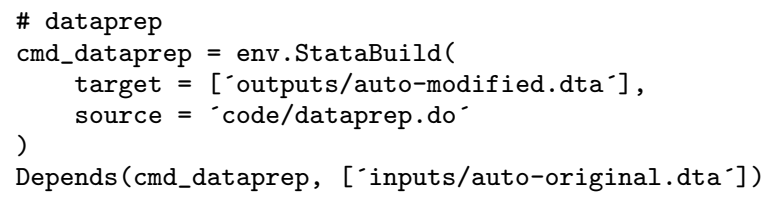

Let's consider first the analysis task. We have given this task the name cmd_analysis 7 The targets are scatterplot.pdf and regressionTable.tex. The source file for this task is analysis.do. The source file is the code we will run to build these targets. The Depends () line lists any dependencies other than the source file ${ }^{8}$ In this case, this is auto-modified.dta. Finally, env.StataBuild tells scons that this is a task for Stata, to be handled in the way defined by the Builder method StataBuild. The essence of the StataBuild Builder method is that scons will build the targets (here, scatterplot.pdf and regressionTable.tex) by running the source file (here, analysis.do) in Stata's batch mode9

7. More precisely, cmd_analysis is a NodeList, where the nodes are the targets. Conveniently, you can use the NodeList elsewhere in the SConstruct to refer to the targets. That is, if we use cmd_analysis elsewhere in this SConstruct, scons will understand this to mean the targets scatterplot.pdf and regressionTable.tex.

8. While not strictly necessary, it is useful to split the source from the other dependencies so there is no ambiguity about what is the code that will be used in the StataBuild environment.

9. Batch mode does not require user input and is often used for automating Stata tasks. See the "Stata batch mode" section of the relevant Getting Started With Stata manual ([GSW] B.5, [GSU] B.3, [GSM] B.3). 
The dataprep task, cmd_dataprep, is similar and we leave parsing it as an exercise for the reader.

We will now use statacons to build our outputs. We will run statacons with the option debug(explain), which adds some helpful screen output that tells us what is going on and why.

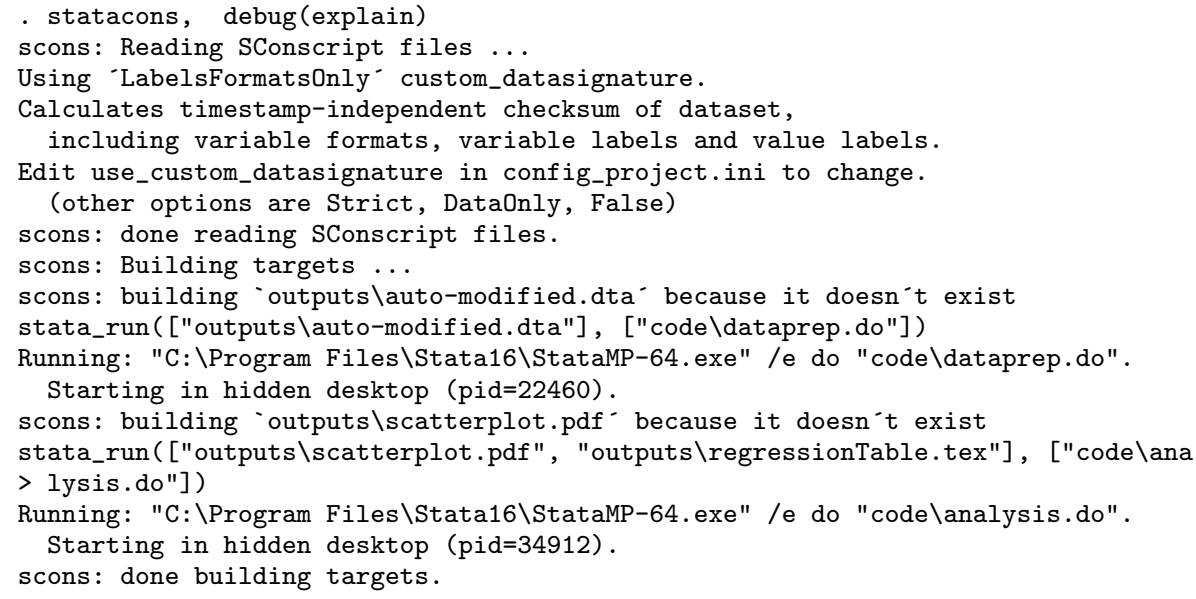

There are a few key lines from the screen output that we will highlight:

scons: building 'outputs\auto-modified.dta' because it doesn't exist

This tells us that scons has noticed that auto-modified.dta does not exist, and must be created. The way it is created is given in the next two lines:

stata_run (["outputs\auto-modified.dta"], ["code\dataprep.do"])

Running: "C:\Program Files \Stata16\StataMP-64.exe" /e do "code\dataprep.do"

The first of these is what is sent to the Stata builder in scons: in words, "build the target auto-modified.dta using the source dataprep.do in the way defined in the Stata builder." The second is what the builder tells the computer to do: run the do-file dataprep. do in batch mode ${ }^{10}$

Having created auto-modified.dta, SCons now notices that its next target, scatterplot.pdf, does not exist, and needs to be built, which it does by running analysis.do in batch mode. SCons then concludes that all targets are up to date, and exits.

Incidentally, note that the order in which the tasks appear in the SConstruct is not important. Even though the SConstruct lists the analysis task first, the logic of the build requires that SCons perform the dataprep task first 11

10. Exactly where the Stata executable is on your machine will differ from user to user. We have written statacons to find it automatically for most standard setups, and allow users to adjust the default in configuration files. We discuss configuration files, by default named config-project.ini and config_local.ini, at greater length in Section 3.4

11. Of course, the way the SConstruct is written affects how easy it is for a human reader to understand it. In some cases, it may be easier to follow if the main outputs are listed first, as we have done here. In other cases, a "chronological" ordering may be preferable. 
Now if we ask statacons to rebuild, it will check whether the dependencies of any target have changed since the previous build.

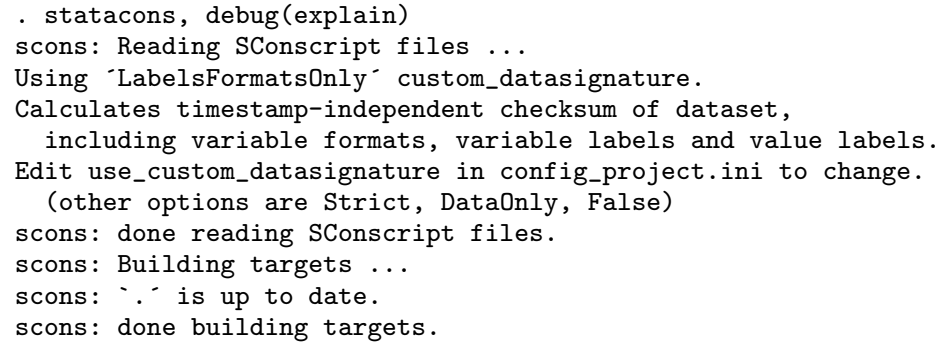

statacons does this by calculating file signatures - a compact string that will not change if the file contents do not change, and almost certainly will change if the file contents change - for each target and dependency, and comparing these signatures to those from the previous build, which it has saved in a database called .sconsign.dblite ${ }^{12}$ The stataconsign command (a Stata call to the SCons command sconsign) will print the content of the.sconsign.dblite.

As an example, here is the the current state of the .sconsign.dblite database for this Introductory Example:

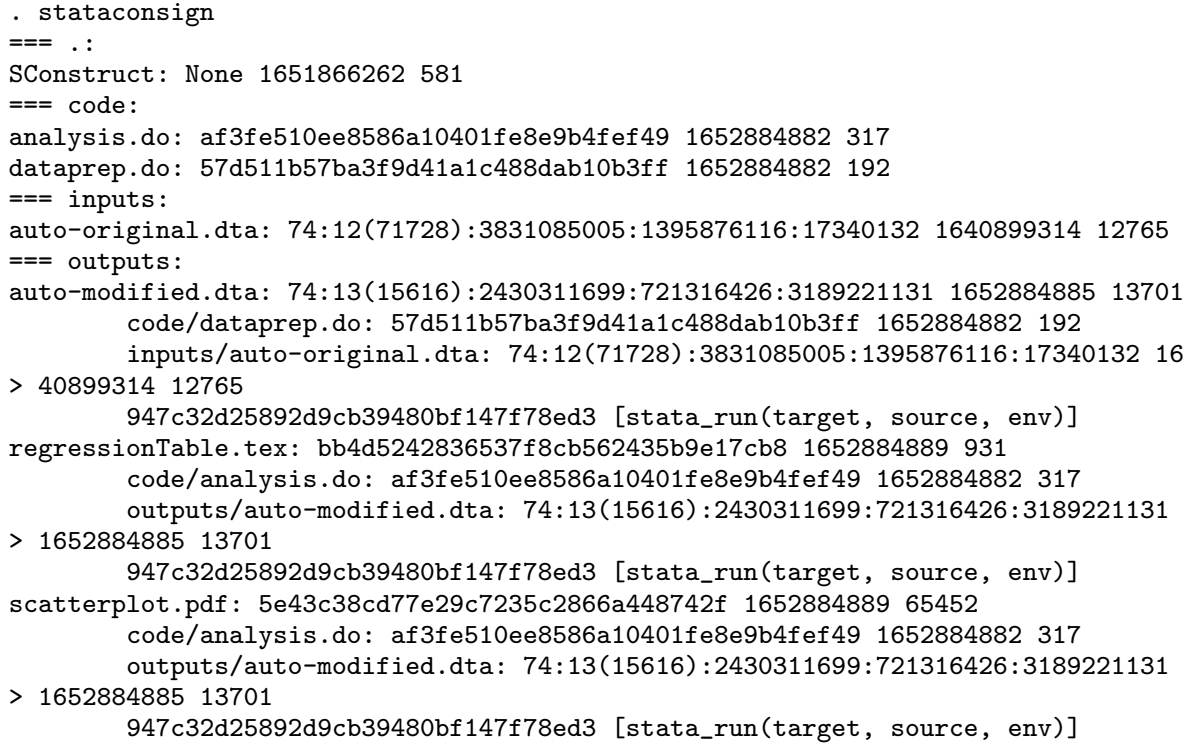

Examining the database provides insight into how SCons and statacons work. For

12. Technically there are different types of functions: "hashes", which provide some cryptographic guarantees, and "checksums", which do not. In the text, we use the more generic term "signature" for all these functions. 
the code (analysis.do and dataprep.do), the database is straightforward. Each is listed with three elements: a signature, a timestamp, and the file length. Similarly, for the input dataset auto-original.dta, the dataset entry consists of the same three items, although the signature is calculated differently, as we discuss in Section 5

For generated files, i.e., files that are built by statacons, the database entries are more complex. As an example, consider the line for auto-modified.dta. Recall that this is the output of the dataprep task. The first line in the database entry for auto-modified.dta has the same structure as those of the code and input data: a signature, a timestamp and the file length. What is different is that the entry for auto-modified.dta has three additional lines: one for each of the two dependencies (auto-original.dta and dataprep.do) and one for the build action stata_run. The lines for the dependencies just replicate their own database entries. The final line is a signature for the build action. These are the three items that SCons needs to consider when deciding whether to rebuild auto-modified.dta. If any of the dependencies or if the build action have changed, SCons will decide to update auto-modified.dta. If none have changed, SCons will know that it does not need to rebuild. The remaining entries are all for derived files and all have the same structure: one line for the file itself, one line for each dependency and one for the build action.

In this case, statacons determines that no dependencies have changed since the last build, so nothing needs to be done.

Now, let's see what statacons does when we make a small modification to analysis . do only. We add a title to the scatterplot, changing

twoway scatter price mpg

to

twoway scatter price mpg, title("Price vs. MPG")

What happens when we run statacons?

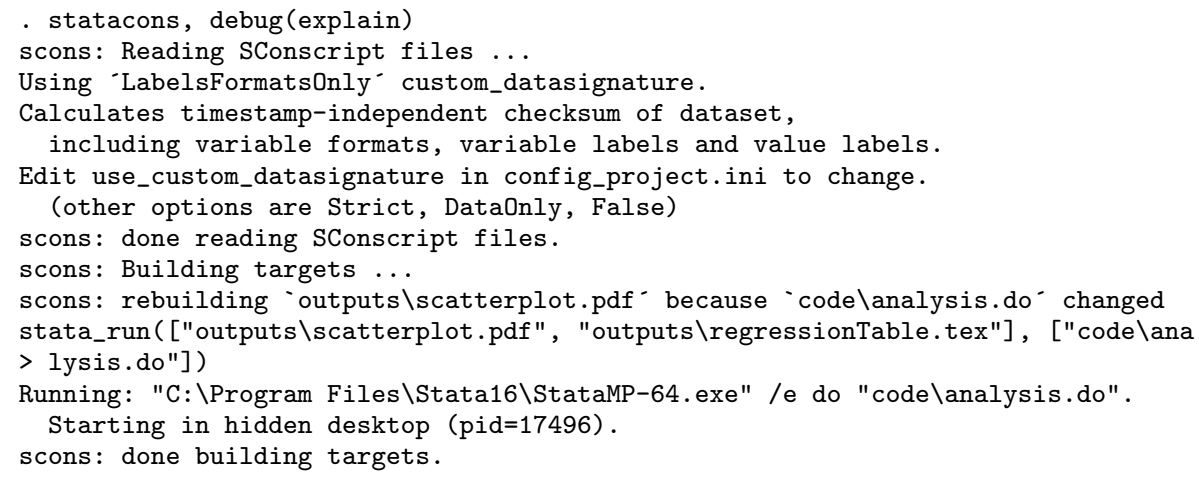

statacons sees that we need to rebuild our target scatterplot.pdf, because its source file analysis.do has changed. statacons also sees that there have been no changes to the dependencies for auto-modified.dta, so it does not need to rebuild that. 
Next, let's explore what happens when we make an edit to dataprep.do. We add a variable mpg_cub, the cube of mpg:

generate $\mathrm{mpg}_{-} \mathrm{cub}=\mathrm{mpg}^{\wedge} 3$

label variable mpg_cub "Mileage (mpg) cubed"

And we rebuild using statacons:

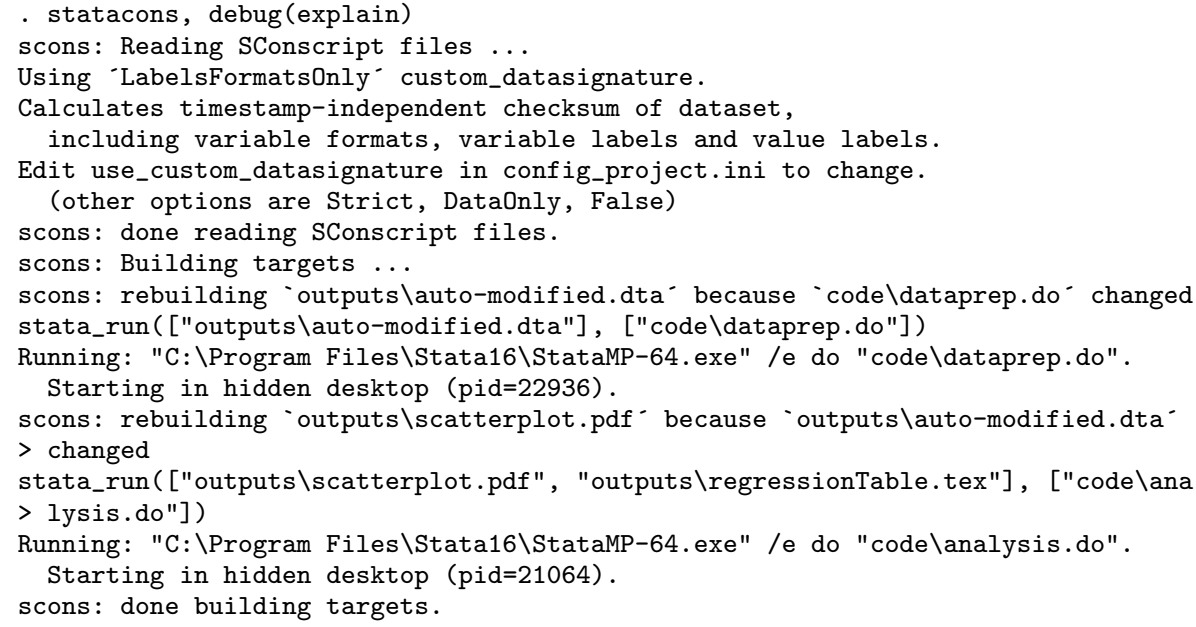

First, statacons sees that dataprep.do, the source for auto_modified.dta, has changed, so auto_modified.dta must be rebuilt. Then, after rebuilding auto_modified.dta, statacons sees that it has changed, and since auto_modified.dta is a dependency for our ultimate targets scatterplot.pdf and regressionTable.tex, these targets must be re-built 13

Finally, let's make a small edit to dataprep. do that does not change the content of auto-modified.dta:

// this is an edit to dataprep.do that does not change auto-modified.dta

The result of rebuilding with statacons may be surprising:

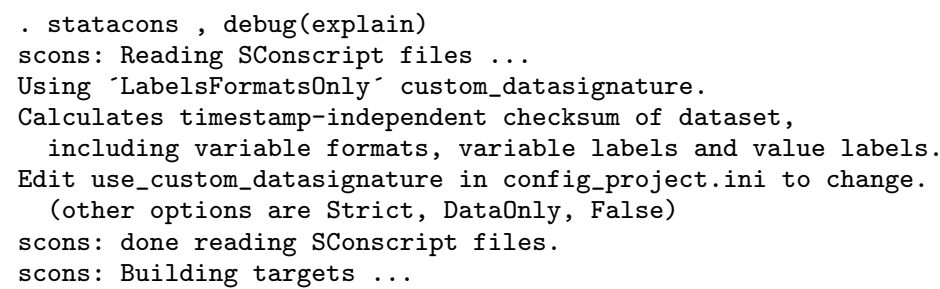

13. Careful readers of the underlying do-files might note that analysis.do does not actually use the variable mpg_cub. However, statacons does not know that - it only sees that a dependency has changed and, since the targets potentially could change, they must be rebuilt. See Section 4 for a strategy to avoid this sort of quasi-false positive. 


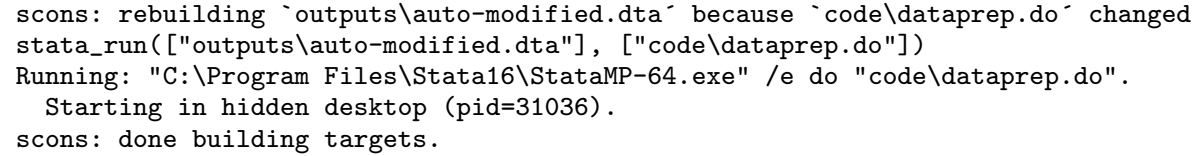

This highlights an important way in which scons, and therefore statacons, differs from the classic and widely used build tool make and many of its derivatives ${ }^{14}$ As we would expect, statacons sees that dataprep.do has changed, so auto-modified.dta must be rebuilt. However, notice that statacons does not rebuild scatterplot.pdf and regressionTable.tex. This is because, while auto-modified.dta has been re-built, nothing about it has changed other than its timestamp. statacons will re-build a target only if a dependency has changed. When deciding whether to re-build scatterplot.pdf and regressionTable.tex, statacons computes a signature of auto-modified.dta that depends only on the content of the dataset, not on its timestamp. Then statacons will check whether this signature has changed since the previous time the target was built. Since none of the content of auto-modified.dta has changed, its signature is unchanged, and statacons sees that there is no need to rebuild any more targets.

\section{Syntax}

The syntax of statacons follows that of scons. For the most commonly used options, we have adapted SCons syntax into a style more familiar to Stata users. These options are listed below. We also allow users to use the standard SCons syntax, although not a mix of the two in the same command 15

The full syntax of scons is too long to replicate here, but can be found in the SCons manual (SCons Development Team 2021a). Here, we present the basic syntax and several of the most useful options, which we have made available in Stata syntax. For options not listed here, the user will need to use standard SCons syntax. More in-depth and comprehensive discussion can be found in the SCons User Guide (SCons Development Team 2021b).

The syntax of statacons is:

$\underline{\text { statacons }}[$ targets $][$, options(values) $]$

The SCons equivalent is:

statacons $[$ option[=value]][targets]

By default, statacons will look in the current directory for an SConstruct file, consider all targets described by that file, and then build or re-build targets when needed.

14. For example, GNU Make: https://www.gnu.org/software/make/.

15. Invoking SCons from a terminal prompt requires SCons syntax. 


\subsection{Selecting targets}

The user can select a single target or a subset of targets by specifying them on the command line. In the example from the previous section, to rebuild only auto-modified.dta but not the other targets, we would use:

- statacons outputs/auto-modified.dta

The SCons equivalent is identical.

Alternatively, the user can edit the SConstruct file to specify the default targets using the Default() function. See the SConstruct Syntax subsection below.

If no targets are specified on the command line and no Default() function given in the SConstruct, then statacons will consider all targets in the SConstruct in the current directory or sub-directories.

\subsection{Options}

\section{Standard scons options}

We discuss several of the most commonly used options. For a complete list of options, see the SCons manual (SCons Development Team 2021a).

clean will clean, i.e., delete, specified targets. Use this option with care, since by default it will remove all targets defined in the SConstruct.

SCons equivalent: -c, --clean, --remove

debug (type[,type...]) will print additional information to the screen to help debug the build process. What information is printed depends on the type or types specified. The most useful type is explain, which will lead statacons to print an explanation for each target it selects to build or re-build - typically, which dependency has changed. Note that explanations are not given for targets that are not built.

SCons equivalent: --debug=type $[$, type...]

directory(directory) change directory to directory before starting build

SCons equivalent: -C directory, --directory=directory

dry_run will put SCons into no execute mode. SCons will attempt to determine what targets it would build and print the commands it would execute, but will not actually execute these commands.

SCons equivalent: -n, --no-exec, --dry-run, --just-print

It is important to note that because SCons by default rebuilds targets only when dependencies have changed, SCons may not be able to determine whether it will need to rebuild "downstream" targets. For example, suppose A.dta is a dependency of B.dta and B.dta is a dependency of C.dta. When A.dta changes, SCons knows 
it must rebuild B.dta. However, since in no execute mode SCons does not actually rebuild B.dta, it does not know whether B.dta will change. As a result, it does not know whether it will have to rebuild C.dta and will not print any commands for rebuilding C.dta. In general, we can think of the default set of commands printed in no execute mode as the smallest set that will be executed.

file(file) or sconstruct(file) allows the user to specify file as the initial SConstruct file for statacons to read. By default, statacons will look for a file named SConstruct in the current directory.

SCons equivalent: -f file,--file=file,--sconstruct=file

help prints the full SCons help message to the screen. The user can define an additional help message in the SConstruct using the Help function, see the SCons manual for details. (statacons, help is distinct from help statacons, as the latter gives the help file for the Stata command, not the SCons program.)

SCons equivalent: $-\mathrm{h},--\mathrm{help}$

q do not print SCons status messages ("quiet")

SCons equivalent: $-Q$

silent do not print messages about SCons actions nor SCons status messages ("silent"). Implies q.

SCons equivalent: -s, --silent, --quiet

tree (type [, type...]) prints a dependency tree, which is useful for debugging or checking on the status of a build, or just for visualizing and understanding the workflow. The part of the tree printed and the format depend on the type or types specified:

all: print the entire tree

derived: print only "derived" (i.e., target) files. Omit files that are not targets, e.g., code or input data.

linedraw: uses unicode characters to draw the tree rather than the default ascii. Some users may find this easier to read on the screen.

status: prints the current status of each item on the tree. This is useful for understanding the status of a build, since it will tell you which files are current ("C"), which files exist ("E"), and several other status categories.

prune: makes the tree easier to read by not repeating dependencies for files that have already been described by the tree. Without this option, tree prints a lot of redundant information. With the prune option invoked, tree will indicate that a file's dependencies have already been listed by enclosing the file name in [square brackets].

SCons equivalent: --tree=type $[$, type...]

We most commonly invoke tree as tree(status, prune). For our introductory 


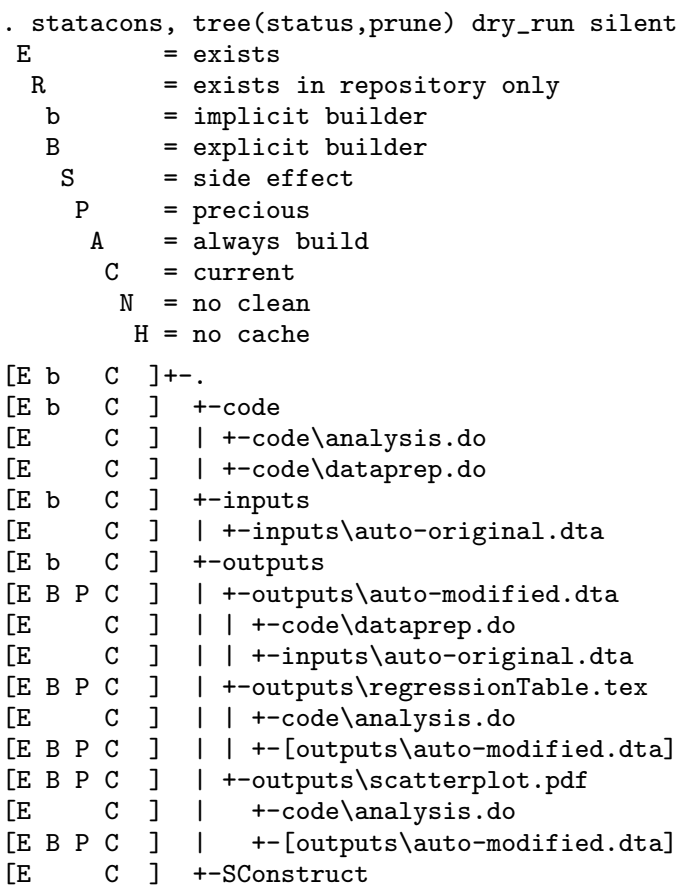

Figure 3: SCons tree from our Introductory Example

example, this produces the output shown in Figure 3.

Exporting the output of tree to a text file ${ }^{16}$ will produce output similar to that of the BITSS / Social Science Reproduction Platform Diagram Builder (BITSS 2020).

\section{Custom statacons options}

All of the options above are standard to SCons. We have written several options specifically for statacons ${ }^{17}$

assume_built("target") will instruct the Stata builder to skip a task if all of its targets are listed and then to mark those targets as up-to-date. This can be a colon-separated list of file patterns.

SCons syntax: --assume-built=" target"

assume_done ("filename.do") will instruct the Stata builder to skip the given do-file

16. For example, in a Unix-like terminal, enter scons -ns --tree=prune > outputFile.txt

17. Standard SCons (i.e., invoked as scons from the command line) will process these commands as long as you have installed the Python package pystatacons and your SConstruct imports pystatacons. SCons-style syntax will be required. 
in the current build, but mark the associated target(s) as up-to-date. This is useful if we know that a target is up to date but SCons does not recognize it as such, for example: (1) if we have just built target.tex by running filename.do directly in Stata rather than through scons or statacons; (2) if we have only made edits that we know will not result in any changes to target.tex, such as adding comments in filename.do. Works even if the task for this do-file includes parameters or a non-do file command. See discussion in Section 6.3. "Limitations,"

SCons syntax: --assume-done="filename. $d o "$

assume_done ("file name1.do:file name2.do[: ... ]") will split by the colon and skip each file. Each component can be a file pattern, as seen in the next example.

SCons syntax: --assume-done="file name1.do:file name2.do[: ... ]"

assume_done $(*)$ will skip all do-files in the current build and mark all their targets as up-to-date.

SCons syntax: --assume-done $=*$

config_file(CONFIG_FILE). By default, statacons will look for config_project.ini and config_local.ini in the same directory as the SConstruct. If these files do not exist or they do not specify several core parameters, such as the location of the Stata executable, defaults based on typical configurations for the user's operating system, version and edition of Stata, will be used. Setting config_file(CONFIG_FILE) will instruct the Stata builder to use CONFIG_FILE as the configuration file. See Section 3.4 for more on default settings and configuration files. The user can specify multiple files as FILE1:FILE2: ...:FILEN. In case of conflicts between files, the file that appears later in FILE1:FILE2: ...:FILEN will take precedence.

SCons syntax: --config-file=CONFIG_FILE

show_config will print the current values of all configuration options, whether chosen by default or specified in a configuration file. statacons, show_config activates the dry_run and silent options, so statacons will not build any files or report anything about the state of the build.

SCons syntax: --show-config

\subsection{SConstruct Syntax}

\section{Basic SConstruct Recipe}

The basic recipe for encoding a target built by Stata into an SConstruct file is:

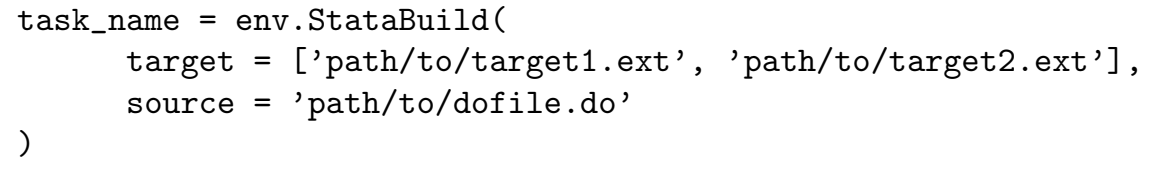




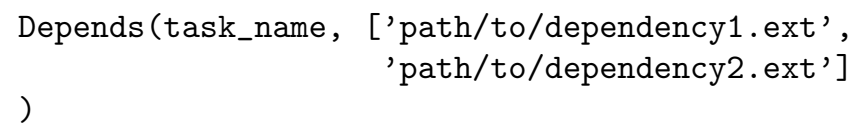

In Section 2, we described each of the elements: builder (here, StataBuild), target, source, and dependencies. target and source are both required 18

\section{Additional Options for SConstruct Recipe}

We have added some options to the basic recipe:

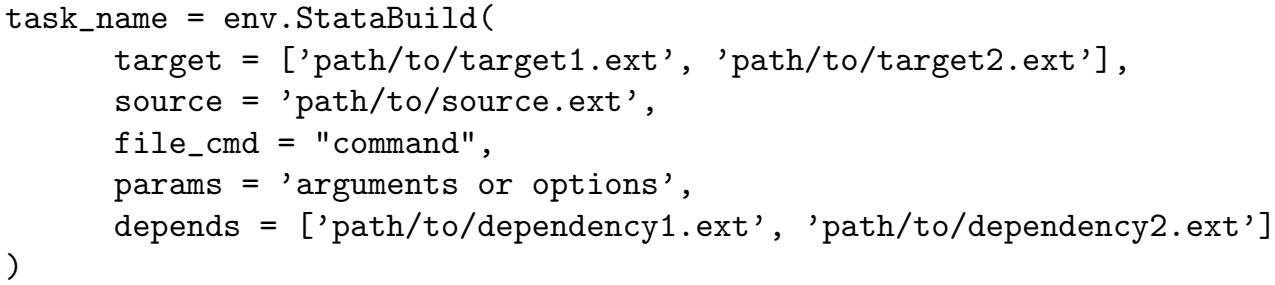

The additional options are as follows:

- file_cmd: the command that SCons should pass to Stata's batch mode. The default is do, but the user can specify anything that Stata can accept as a command, e.g., dyndoc, markdown, etc.

- params: arguments or options that should follow the source in the call to Stata batch mode. For example, a call to markdown might specify

params $=$ ', saving (myfile.html) replace'

- depends: an alternative to using the Depends() function

We include several additional examples of these options in our web tutorial 19

\section{Defining Lists of Files in SConstructs}

As projects grow in size, it may become unwieldy to write out long lists of dependencies. Furthermore, if different targets have overlapping sets of dependencies, writing the same dependencies in different places is inefficient and can lead to errors. We can do better by defining variables in our SConscript files using standard Python syntax and some special SCons functions. As a preview of the Applied Example in Section 4 below, we illustrate two of these methods.

18. For managing tasks that do not build specific targets, see 'Alias and Phony Targets' below. 19. https://bquistorff.github.io/statacons/swc.html, especially the "Paramters" lesson. 
One of the targets, ctyQtrWages .dta, uses as inputs five Excel files named wagedata1.xlsx, ..., wagedata5.xlsx. We could write these out explicitly in the Depends() line, but instead we will demonstrate two alternatives. The first is the SCons Glob() function:

wageDataFiles $=$ Glob ("inputs/wagedata[1-5].xlsx")

Depends (wage, wageDataFiles)

where [1-5] means "any element of the sequence 1, 2, .., 5."20 SCons will search the directory inputs for files matching the pattern and will define wageDataFiles to be the set of files that match.

A more flexible alternative is to use Python's "list comprehension" method as follows ${ }^{21}$

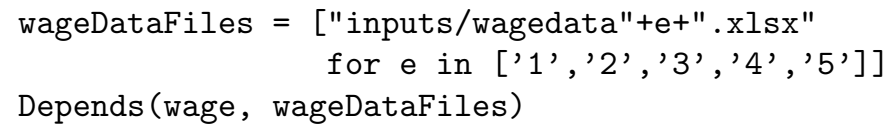

While this method is not as concise as Glob(), it has a few advantages. First, it allows for more complex structures and patterns. For example, a sequence of ISO country codes would begin 'AFG', 'ALA', 'ALB'. Second, it can define lists of targets that may not yet exist, while Glob() is limited to matching existing files. Third, it is more precise. As a concrete example, suppose the file wagedata1.xlsx is missing from the dataprep/inputs directory. If we write out all our dependencies explicitly or use the list comprehension method above, SCons will return an error, which is useful. If we use Glob ("inputs/wagedata[1-5] .xlsx"), SCons will think that the dependencies are wagedata2.xlsx, ..., wagedata5.xlsx, i.e., the files that are present, and will proceed with the build 22

See the companion web tutoria ${ }^{23}$ for more examples of using Python coding to write more concise SConstructs.

\section{SConstruct Functions}

We discuss only a few of the more useful functions available in SConstruct files. See the SCons manual or the SCons User Guide for a comprehensive list and more details on implementation. We also note a few additional options that the pystatacons package provides.

20. Glob() allows unix shell pattern matching, i.e., * (match everything), ? (match single character), [seq] (match any singe character in the sequence seq), and !seq (match any single character not in the sequence seq). See the SCons User Guide for more details.

21. See https://docs.python.org/3/tutorial/datastructures.html for more information on list comprehension and other Python data structures.

22. Despite our concerns about Glob(), we have used it in our Applied Example for the sake of providing an example. See the definition of the industryCompFiles variable in dataprep/SConstruct.

23. https://bquistorff.github.io/statacons/swc.html, especially the "Variables" lesson. 
Note that some of these functions (e.g., SetOption()), will require the use of *nixlike standard SCons syntax rather than our adaptation to Stata syntax.

AlwaysBuild(target [, target...]): instructs SCons that the given targets should always be rebuilt when they are specified, even if they are up to date. Note that a target must be specified either in the command line or as part of the default set that is, AlwaysBuild() neither implies nor is implied by Default().

Default(): allows the user to specify which targets SCons will examine by default. In our introductory example, we would set a default of rebuilding only auto-modified.dta but not the other targets by adding

\section{Default ('outputs/auto-modified.dta')}

to the SConstruct. If Default() is not specified, SCons will examine all targets in current directory or subdirectories unless otherwise specified on the command line.

Decider (function): determine how SCons will decide whether a target is up to date.

"content": the default. For each dependency of a target, SCons will calculate a signature and compare the result against the last time the target was built ${ }^{24}$ This means that a target will be rebuilt only if one if its dependencies have changed. So, suppose A.dta is a dependency of B.dta and B.dta is a dependency of C.dta. Now, suppose that A.dta changes, but when B.dta is rebuilt, it does not change. Even though B.dta is newer than C.dta, SCons will not rebuild C.dta.

"timestamp-newer": SCons will rebuild a target if any of its dependencies are newer than the target itself (similar to the classic build tool make). Suppose A.dta is a dependency of B.dta and B.dta is a dependency of C.dta. Now, suppose that A.dta changes, but when B.dta is rebuilt, it does not change. Even though B.dta has not changed, it is newer than C.dta, so SCons will rebuild C.dta. Because it does take time for SCons to calculate signatures, timestamp-newer may be faster despite some unnecessary rebuilds if there are relatively many input files (and therefore many signatures to calculate) and relatively low computation time.

"timestamp-match": SCons will rebuild a target if any of its dependencies have a timestamp different (newer or older) than the last time the target was built. If all dependencies' timestamps are the same as the last time the target was built, the target will be considered up to date and will not be rebuilt.

"content-timestamp": rebuild a target only if a dependency's timestamp and signature have changed since the last time the target was rebuilt, that dependency is considered up to date. This is similar to content, except it skips checking signatures for dependencies whose timestamps are unchanged and therefore may run faster.

"content-timestamp-newer": rebuild a target only if a dependency's signature

24. Rather than recalculating the signature, SCons will save time by using a cached value of the signature if (1) a cached value is available from previous builds, (2) the modification timestamp has not changed, and (3) the timestamp is at least as old in seconds as the max-drift parameter (default is two days, but is configurable). 
has changed since the last time the target was rebuilt and any of its dependencies are newer than the target itself. Similar to the assume_done and assume_built command-line options defined above, this means that SCons will not re-run Stata code that has already been run outside of SCons. Unlike assume_done and assume_built, it only needs to be specified once in the SConstruct, not specified at the command line each time. "content-timestamp-newer" is defined by pystatacons. To use it, include Decider (pystatacons.decider_str_lookup ["content-timestamp-newer"]) in your SConstruct after pystatacons has been imported.

Ignore (target, dependency): tell SCons to ignore the file dependency when deciding whether to rebuild target.

Ignore (directory, target): remove target from the set of default targets. The first argument of Ignore() must be the directory where target would be rebuilt. SCons will still build target if (1) target is specified on the command line or (2) target is a dependency of another target and needs to be rebuilt.

Requires (target, prerequisite): specify that target should be built after prerequisite even if prerequisite is not a dependency for target. This is useful if the user wishes to impose some order on the build even if that order is not required by the build logic. For example, the user may want to move longer-running tasks later in the build so she can inspect the output of shorter-running tasks while the others run.

SetOption(name, value): allows some command-line options to be set in the SConstruct. For example, SetOption('silent',1) is equivalent to silent on the command line. See the User Manual for the options that can be set by SetOption(). The command line over-rides values set through SetOption().

\section{Alias and Phony Targets}

The Alias () function allows users to give names to targets or groups of targets. This can make it easier to build only a subset of targets, or to refer to groups of targets in an SConstruct.

In our Introductory Example, suppose we wanted a shorthand for the dataprep process so that, rather than call outputs/auto-modified.dta from the command line, we could use just dataprep which is shorter and easier to remember. We would code:

dataprep_Targets $=$ ['outputs/auto-modified.dta']

Alias ('dataprep', dataprep_Targets)

into our SConstruct and then, if we wanted to build only the dataprep targets, call

statacons dataprep

instead of

statacons outputs/auto-modified.dta

For additional uses of Alias () to create a convenient way to reference groups of files, 
see SConstructWithAlias and other SConstructs from the Introductory Example ${ }^{25}$

A second use of Alias () is to incorporate commands associated with a project that do not build files: configuring the local environment, communicating with a server (e.g., publishing a package), or displaying information in the terminal. For convenience, these can be stored in your SConstruct by assigning them to "Phony" targets (targets that are not real files) using Alias. For example, if we put the following line in our SConstruct, we could run the shell command dir whenever we run SCons with the target show_dir.

env.AlwaysBuild(env.Alias("show_dir", action="dir"))

As show_dir is not a real target with an associated build recipe, we encase Alias() in AlwaysBuild(). The command dir could be replaced with a Python function (potentially user-defined) to allow for more options, as we will see in Section 6.1.

\subsection{Default Settings and Configuration Files}

We have incorporated what we think are sensible defaults into statacons. These include:

- finding the Stata executable automatically by searching the user's PATH variable and other default locations;

- using our custom complete_datasignature.ado to compute signatures for .dta that depend only on their content, not their embedded timestamp. (See discussion in Section 5; ;

- deleting the batch-mode generated log-files after a do-file is successfully executed in batch mode, but retaining it in the default directory if the do-file fails.

By setting these defaults, we reduce the need for users to spend time figuring out how to configure the tool, which we suspect has been a barrier to adoption of build tools previously. However, some users may wish to choose other options or have unusual setups. Therefore, we allow users to change their configurations in two files, config_project.ini (for settings common to all users in a given project) and config_local.ini (for user-specific settings). We provide templates for both with suggested values in the project files installed with statacons. In case of conflicts between config_project.ini and config_local.ini, config_local.ini will take precedence.

These configuration files follow a simplified INI format readable by Python's standard library 26 . They are simple text files that are easily read and edited. Each file stores key-value pairs organized under one or more headers.

[header1]

25. https://github.com/bquistorff/statacons/raw/main/examples/stataconsIntro.zip 26. See https://docs.python.org/3/library/configparser.html for full details 
key1: value 1

key2: value 2

[header2]

key3: value 3

As an example, the user may wish to retain the log-files created by batch-mode Stata in a folder called logs, which can be done in config_local.ini with

[SCons]

success_batch_log_dir: ./logs/

As a second example, by default SCons operates relative to the directory where the SConstruct is found, so this is the directory from which Stata will run in batch mode. This is a reasonable default, but some users may wish for Stata to start in a different directory. We allow this through a stata_chdir option in config_project.ini. See the config_project.ini file provided with this package for the available options.

We provide some additional examples of the use of configuration files elsewhere in this paper, such as telling SCons where to find shared folders in Dropbox. Users can specify other configuration files using the config_file() option listed in the Custom statacons options section above. As mentioned there, in the case of conflicts between config files, the file listed later in config_file() will take precedence.

\subsection{Other Advanced Features}

We conclude this section by briefly mentioning a few advanced features of SCons. We have additional discussion and examples in the Appendices. This is not a complete list nor a comprehensive guide; rather, we aim to familiarize readers with some concepts and vocabulary they may encounter while using the tool.

The first is the SConscript, which allows users to split a long SConstruct into more manageable parts, i.e., a hierarchical build. See Appendix A.1.

This second is SCons's ability to manage parallel builds, conducting tasks simultaneously rather than sequentially when the logic of the workflow permits. SCons parallel builds are well-suited for a relatively small number of relatively slow tasks. For tasks with a relatively high number of relatively quick tasks, like bootstrapping or simulation, the approach of parallel (Vega Yon and Quistorff 2019) is preferable. We provide an application of parallel builds to our Applied Example in Appendix A.2. Parallel builds are currently only available in scons (i.e., from a terminal), not in statacons.

The third is the SCons cache, which allows storing and sharing of derived files. We describe the use of the cache in Appendix B in the context of collaborative workflows. 


\section{Applied Example}

This section describes the use of statacons in an empirical project (Shumway and Wilson 2021). Key build scripts and do-files are provided in appliedExample.zip. posted to our repository ${ }^{27}$

For our purposes, this project has two main parts: dataprep, in which raw data are cleaned and merged, and new variables are created for analysis; and analysis, in which the analysis of these data is carried out. The value of a build tool is apparent from Figures 4, 5, 6, and 7, rather than trying to remember all these interdependencies, we encode them in SConstructs. Consulting an SConstruct and the output of tree() is a more reliable way to document interdependencies, to orient a new collaborator, remind the user's future self of how a project works after some time away, trace errors, etc., than depending on memory. The SConstructs and output of tree for both the dataprep and analysis tasks are provided in appliedExample.zip on the project repository.

We have separated the two main parts of the project, data cleaning and data analysis, into two directories, each with its own SConstruct. These two directories are linked by a third, which we have called transferDataprep. The phony target post_outputs in the dataprep SConstruct copies the main outputs from dataprep/outputs to transferDataprep, while the phony target pull_inputs in the analysis SConstruct copies these from transferDataprep to analysis/inputs. See Section 6.1 for more details and an extension to shared files.

One of the estimation procedures, the dynamic treatment effects estimator of Sun and Abraham (2021), takes over two hours to run even on a fast desktop. We have written our code and SConstruct to avoid repeating this estimation when it is not necessary. The relevant section of the SConstruct is shown in Figure 8 along with a diagram.

First, this task uses only a subset of the variables and observations in judgePanel.dta. While we do need to re-run the estimation if the data used have changed, we would not want to re-run the estimation if the only variables or observations to change were not used in this task. The first task, sunAbrahamSelect, creates sunAbrahamEstimationSample.dta, which keeps only the variables and observations of judgePanel.dta needed for this task. So, suppose judgePanel.dta has changed. SCons will rebuild sunAbrahamEstimationSample.dta. If sunAbrahamEstimationSample.dta changes, then SCons will go on to the estimation step in sunAbrahamEst, since sunAbrahamEstimationSample.dta is the dependency for that task. However, if sunAbrahamEstimationSample.dta has not changed, then SCons will not repeat sunAbrahamEst.

Similarly, the ultimate outputs are two graphs of estimated treatment effects. Suppose we wanted to change some aspect of the graphs, e.g., the axis titles. We would not want to have to repeat the full estimation. On the other hand, we do want to re-create these graphs whenever the underlying estimates change. We handle this by separating the estimation and the creation of graphs into distinct steps. The task sunAbrahamEst

27. https://github.com/bquistorff/statacons/raw/main/examples/appliedExample.zip 


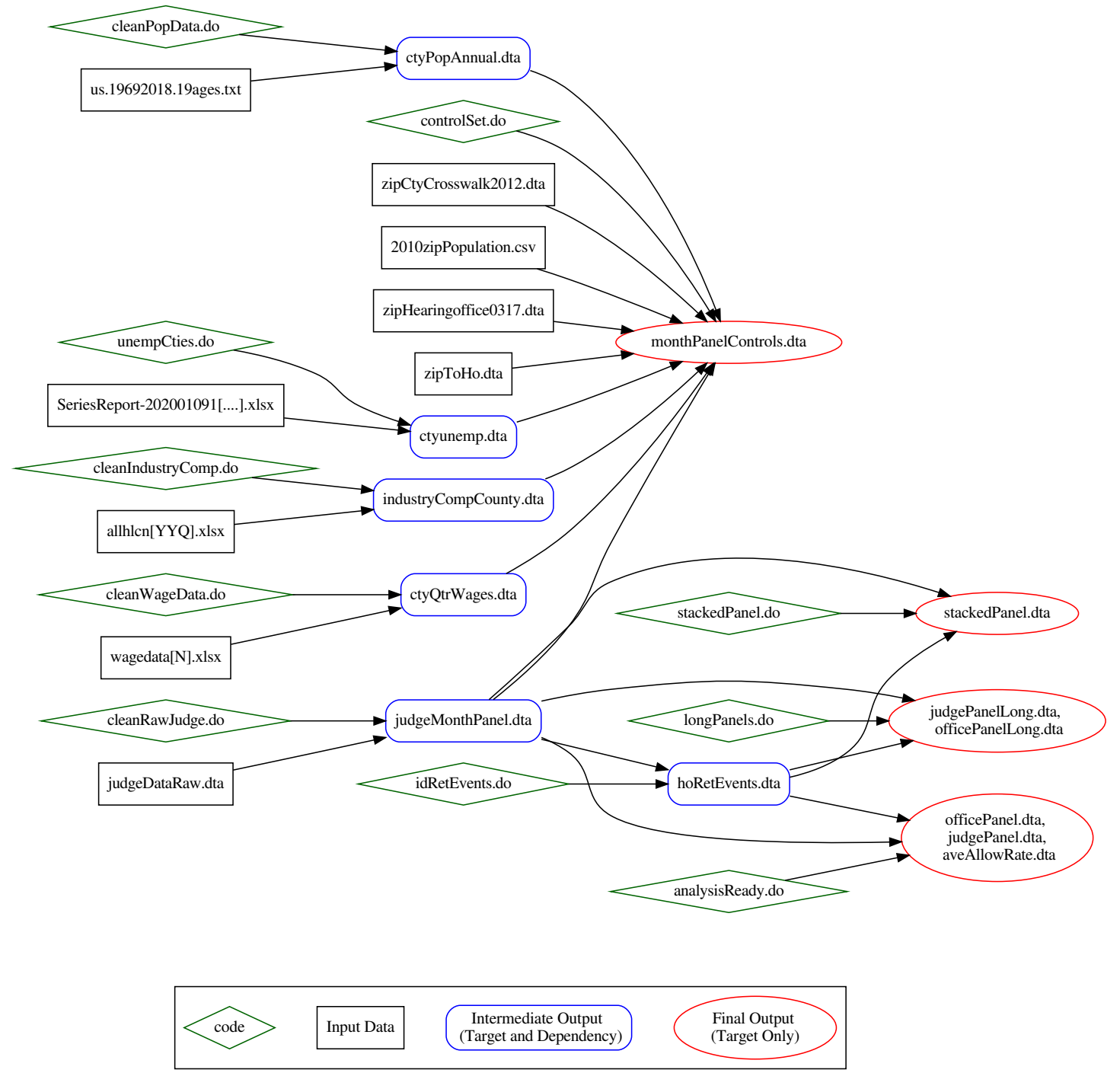

Notes: brackets represent a series of similarly-named files, e.g., wagedata[N] .xlsx represents wagedata1.xlsx, ..., wagedata5.xlsx. We have omitted file paths for brevity.

Figure 4: Workflow for Applied Example: Dataprep 


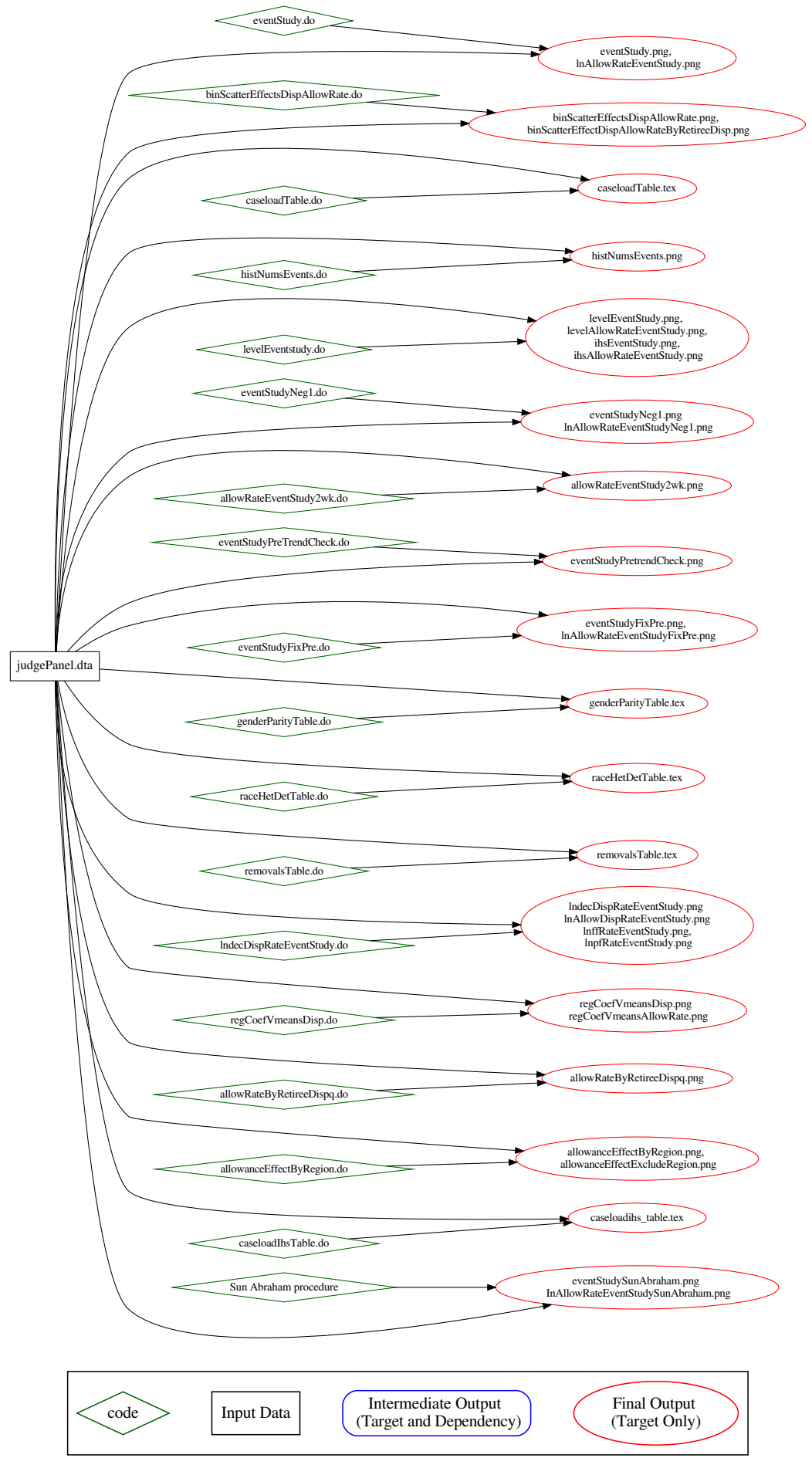

Notes: We have omitted file paths for brevity. The workflow for the Sun-Abraham event study procedure is simplified, see Figure 8 for the complete workflow.

Figure 5: Workflow for Applied Example: Analysis (Part 1) 


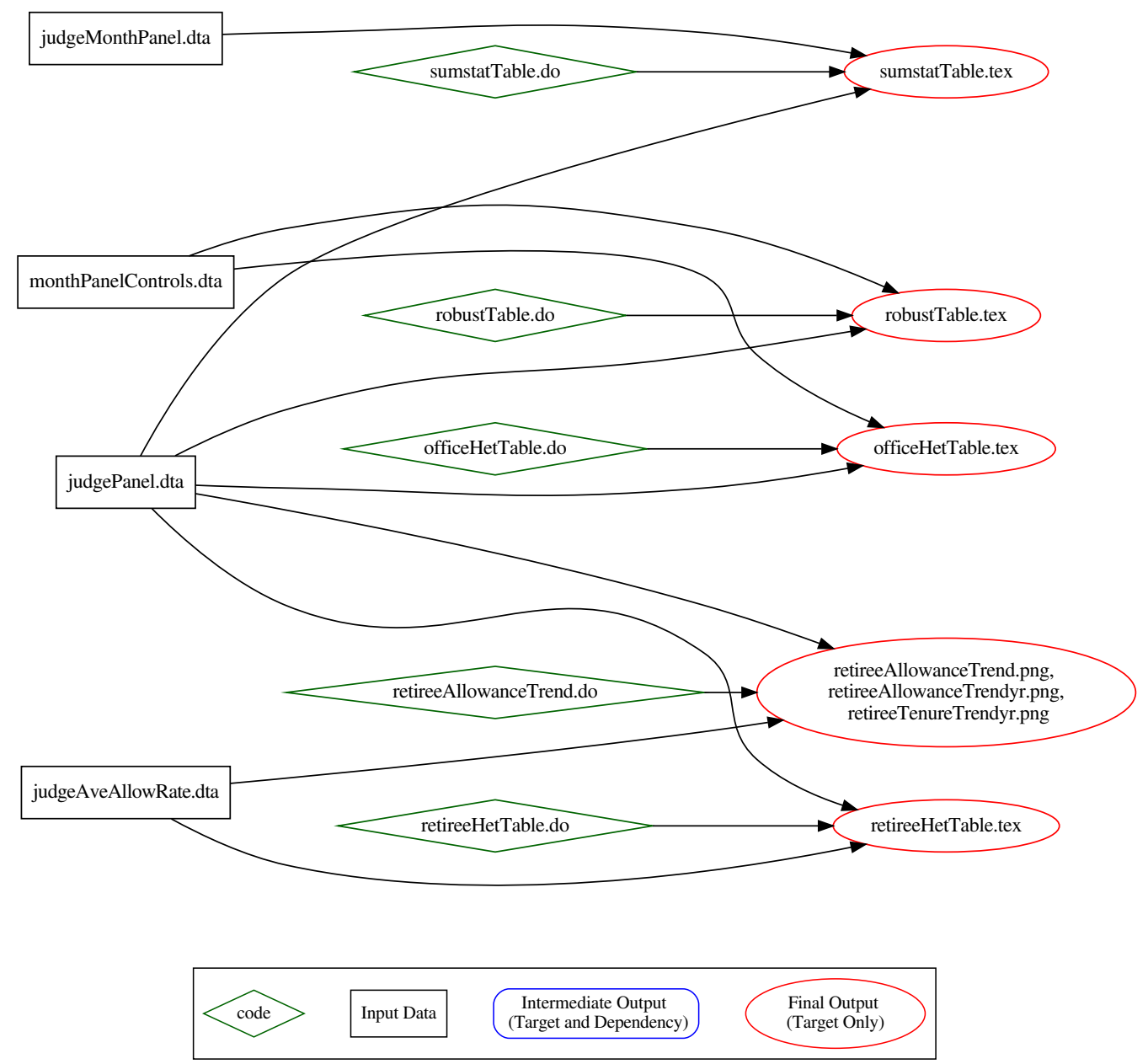

Notes: We have omitted file paths for brevity.

Figure 6: Workflow for Applied Example: Analysis (Part 2) 


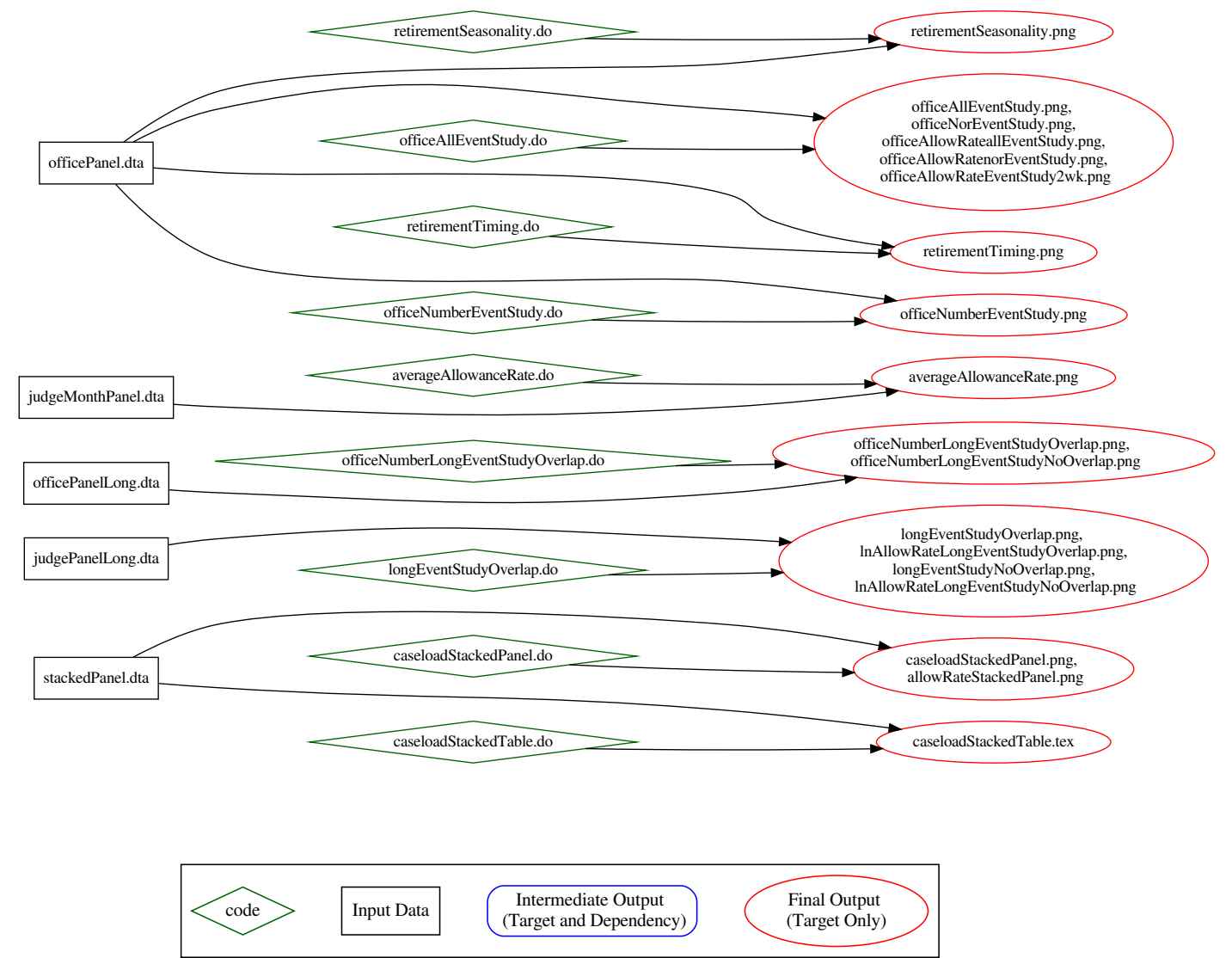

Notes: We have omitted file paths for brevity.

Figure 7: Workflow for Applied Example: Analysis (Part 3) 

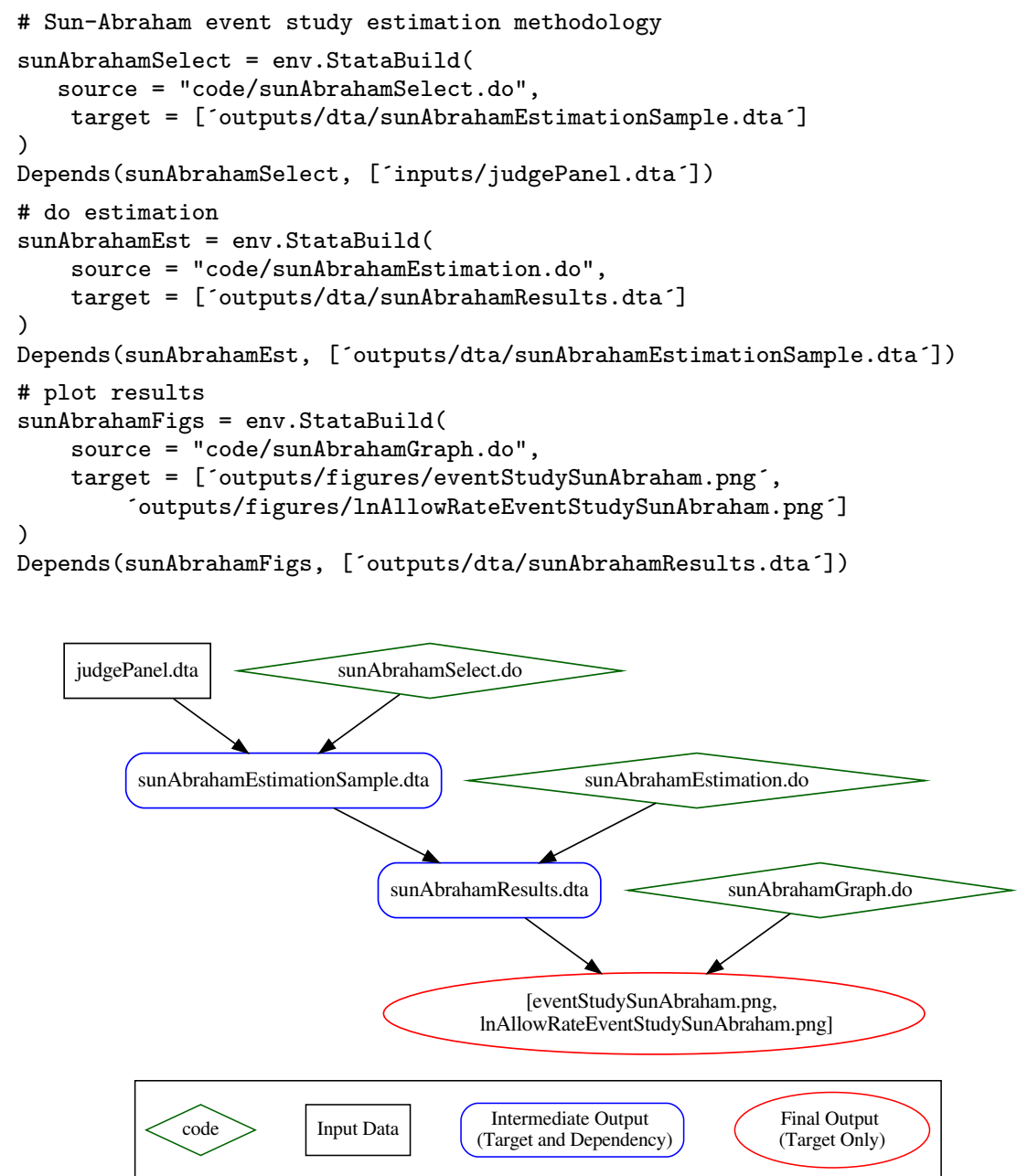

Figure 8: Separation of Concerns in Applied Example 
handles the estimation, saving the estimation results in sunAbrahamResults.dta ${ }^{28}$ Then the task sunAbrahamFigs handles producing the graphs. If we edit sunAbrahamGraphs. do to change some aspect of the graphs, SCons will not see any need to rebuild sunAbrahamEst. On the other hand, if something about the estimation changes such that sunAbrahamResults .dta changes, SCons will automatically rebuild the graphs.

This division of tasks is known as separation of concerns in computer science and is in our view a key distinction between working with a build tool and working in the literate programming paradigm described in Section 6.2. The virtue of literate programming is that everything is in one piece of code, which is certainly convenient and simple. A build tool workflow in which discrete tasks are separated will save computation time. Of course, there is a cost to creating separate do-files, so this tradeoff may not be worthwhile for simple, quick tasks.

Finally, we can further reduce computation time by using SCons's ability to manage parallel builds. We discuss this in Section A.2 of the Appendix.

\section{Technical Details}

We note here some technical details of how our package works.

First, as we have discussed above, SCons's default behavior is to look for changes in the content of dependencies when deciding whether to rebuild a target. SCons does this by computing a signature of each dependency. However, because Stata embeds a timestamp in .dta files, .dta files created at two different times will have different signatures even if the contents are identical. This will lead to unnecessary rebuilds of target files. Stata's datasignature command can create signatures that depend only on the values of the data in the .dta file, not the timestamp, but datasignature signatures do not depend on variable labels or value labels.

We create an ado program complete_datasignature.ado that augments Stata's datasignature with a signature of the dataset's metadata (variable formats, variable and value labels, notes and characteristics) but not the timestamp. We then patch SCons so that it uses our complete_datasignature.ado instead of its default signature algorithm. In the config_project.ini file, the user has several options:

- Strict: include data and all metadata in the signature (variable formats, variable and value labels, notes and characteristics)

- LabelsFormatsOnly: include data, variable formats, and variable and value labels in the signature (do not include dataset labels, notes, characteristics)

28. Here we have saved estimation results into a .dta dataset. An alternative is to use Stata's estimates save command to save results to a .ster file. The user-written estwrite and estread (Jann 2005) improve on estimates save by allowing users to store multiple estimation results in a single .sters file and then refer to individual estimates by model name. Note that as of version 1.2.5, estwrite has an option reproducible that will eliminate the timestamps previously embedded in .sters files. As of this writing, . ster files produced by estimates save contain an embedded timestamp. 
- DataOnly: use Stata's standard datasignature, which depends on data only (does not depend on any metadata or the embedded timestamp)

- False: use SCons's default signature, which will depend on the embedded timestamp

In addition, we provide some control over the use of datasignature's fast mode. The fast mode speeds up the calculation of the file signature, but is not machineindependent: users on different machines may produce different signatures. This is not so important for solo users working on a single machine, but for collaborative workflows (or a single user working on different machines) that save time by sharing built files in a common directory, using the fast option may lead statacons to incorrectly believe that a build is out of date. We make fast the default unless the CacheDir() option is specified (indicating a collaborative workflow, see Appendix B), in which case the machine-independent but slower method is the default. Users can override these defaults, for example to use the slow option for a solo project, through the dta_sig_mode option in the config_project.ini configuration file.

Our next set of issues deals with SCons directing Stata to run in batch mode. First, SCons needs to know if the do file finished successfully to know if targets should be considered built. This would typically be communicated via the program's exit status, but Stata always returns a normal exit even if the script it runs encountered an error. Our Stata builder therefore parses the end of the Stata batch-mode log file to see if there was error and communicate this back to SCons. This requires that batch-mode Stata produce plain-text log-files, so we do not allow the user to change the options with which batch-mode Stata is called.

Second, Stata typically uses the do-file name as the name of the batch-mode log-file, but we may need to change this for a variety of reasons. If two commands would result in the same log file name (e.g., we call the same do-file with different parameters or we have multiple non-do commands), then they can't be run in parallel because Stata will fail to start when the second instance can't open the log file. Therefore, for all commands aside from plain do commands (with no parameters), we have Stata run a generated do-file that then runs the user's command. We name this generated file so that its resulting log file will be unique (we take the original command's typical log-file name and append a short hash of the text of the command).

Finally, although SCons is a Python tool, and Stata 16+ has a Python environment, several hurdles need to be overcome to use SCons from inside Stata. First, Stata sets a handler for several program "signals" for Python at startup. Later, SCons temporarily sets new signal handlers. When SCons is done, though, it tries to reinstate the original handlers in a way that does not deal correctly with the handlers Stata set from outside Python. We therefore patch the SCons function that reinstates the signal handlers to deal with this case and avoid a crash. Second, Stata changes the stdout and stderr communication channels for the Python process at startup. Later, Scons temporarily changes these, but does not restore them correctly when finished. We patch this restore function so that output can continue to be displayed in the Stata window. Third, 
Stata's Python environment is kept intact between Python script calls. SCons, however, is typically only able to be called from a clean environment. We therefore remove references to SCons modules before starting another run.

\section{Extensions, Alternative Approaches and Limitations}

\subsection{Extensions}

There are several ways to add to the basic functions we have described to this point. In increasing order of difficulty, these are: use additional build tools already supplied with SCons; use built-in SCons commands; execute Python commands and utilities; add user-written SCons build tools; write a new builder. We provide a few examples of the first three, and provide references for those interested in the last two.

\section{Additional Built-in Build Tools: Compiling ${ }^{A T} T_{E} X$ with SCons}

SCons comes with a builder for $\mathrm{AT}_{\mathrm{E}} \mathrm{X}$ documents. As long as the user has a $\mathrm{T}_{\mathrm{E}} \mathrm{X}$ distribution (e.g., TexLive, MiKTeX, etc.) installed and known to the system, this will work more or less out of the box. Conveniently, SCons will automatically scan the .tex file for dependencies, so it is not necessary to code dependencies explicitly into the SConstruct. By default, these implicit dependencies include .tex files that have been \include\{\}ed or \imported\{\}ed, graphics files (.pdf, .eps, etc.) included through $\backslash$ includegraphics \{\} , .bib files, among others. The user may need to adjust some construction variables to get the document to compile in the desired way. In Appendix C. we add compiling a .tex file into a pdf to our Introductory Example.

All tools built into SCons are listed in the SCons User Guide 29

\section{Built-in SCons Commands: “Install” (Copy) Built Files Across Directories}

SCons has several built-in utilities that can be convenient tools. See Sections 11 and 12 of the SCons User Guide for a full list. One simple and especially useful tool, Install(), copies files across directories. We use Install () extensively in the code for this paper 30

First, in our Introductory Example, we use Install() to post our table, graph and compiled PDF to Overleaf for use in this paper. We need to tell statacons where it should copy these files, i.e., the Overleaf directory. In general, Overleaf files will reside in Dropbox/apps/Overleaf/ProjectName, where in this case our ProjectName is SJ-BuildTool. If there is only one user, or if all users have the same path to Dropbox 31 we can define this directly in the SConstruct. For a Windows user, this would be:

29. As of Version 4.3.0 (December 2021), this is "Appendix C. Tools,", https://scons.org/doc/production/HTML/scons-user/apc.html.

30. All code is available on our project website and the project files included with statacons contain templates for the configuration files discussed below, with extensive examples.

31. For example through a symbolic link. 
\# define Overleaf directory

overleaf_dir = 'C:/Users/UserName/Dropbox/apps/Overleaf/SJ-BuildTool'

If there are multiple users with different paths to Dropbox, each path must 32 be defined in the user's own config_local.ini configuration file:

\# define Overleaf directory

overleaf_dir: C:/Users/UserName/Dropbox/apps/Overleaf/SJ-BuildTool

and then, in the SConstruct, we read in this variable:

\# read in overleaf directory from config_local.ini

overleaf_dir = env['CONFIG']['Project'] ['overleaf_dir']

Having obtained the target location, we can add our Install() command to the SConstruct:

\# transfer files to overleaf

env.Install (overleaf_dir, [cmd_analysis, pdf_output])

env.Alias ('post_to_overleaf', overleaf_dir)

Install() takes two arguments: the first is the directory to which the files should be copied; the second is the list of files to be copied. Here, the first argument is the path to the Overleaf folder, as defined in the overleaf_dir variable. The second argument, [cmd_analysis, pdf_output], tells SCons to copy the targets of the tasks cmd_analysis and pdf_output. Finally, in the last line, we use Alias() to give this task a convenient name. Now we can post our output files to overleaf with

statacons post_to_overleaf

Our second application of Install() is in our Applied Example ${ }^{33}$ There are two components to this project, dataprep and analysis. We want the outputs of dataprep to become inputs for analysis. A single user working alone could simply write code in analysis to use output files from dataprep directly, or could write a single Install() task in the dataprep SConstruct to push from dataprep/outputs to analysis/inputs, or in the analysis SConstruct to pull from dataprep/outputs to analysis/inputs. However, these strategies may not work well in a collaborative project, since a user in analysis would not want datasets to be changed without warning, and a user in dataprep would not want work in progress to be pulled into analysis. At the cost of slightly increasing complication, we set up a more deliberate workflow.

32. Adept Python coders may be able to program their way around this in the SConstruct by accessing the operating system environment. As we note in the Conclusion, we encourage users who develop enhancements to statacons to make them available publicly, and we provide a project Wiki to host.

33. Code posted at https://github.com/bquistorff/statacons/raw/main/examples/appliedExample.zip 
First, we add a transferDataprep directory on the same level as the dataprep and analysis directories.

Second, we add an Install() task to the dataprep SConstruct that the user can select to push finished outputs from dataprep/outputs to transferDataprep.

\# post outputs to ../transferDataPrep folder

env.Install ('../transferDataPrep', outputs)

env.Alias ('postOutputs', '../transferDataPrep')

To avoid transferring incomplete work, we want the postOutputs task to run only when it is explicitly called (statacons postOutputs at the Stata prompt or scons postOutputs in a terminal). In this case, postOutputs does not run automatically because its "target", the transferDataPrep directory, is not in the dataprep directory - by default, SCons will look for targets only inside the SConstruct's directory and subdirectories.

However, if the target directory is in dataprep or its subdirectories, we will need to remove postoutputs from the default targets. There are two ways to do this. The first is to set the Default() function explicitly and not include the transfer task among the defaults. This may be cumbersome if there are many targets. The second way is to use the Ignore() function in the SConstruct to tell SCons not to build postOutputs unless explicitly called from the command line. The code in the SConstruct would be

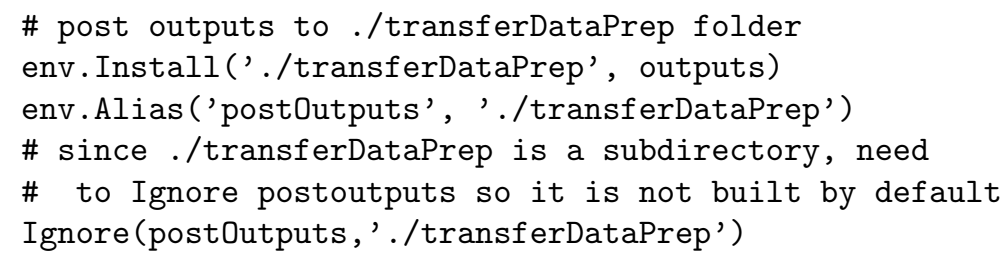

Third and finally, we set up a similar task in the analysis SConstruct to pull data from transferDataprep into analysis/inputs. In this example, we demonstrate the use of Python utilities, here shutil.copytree ${ }^{34}$

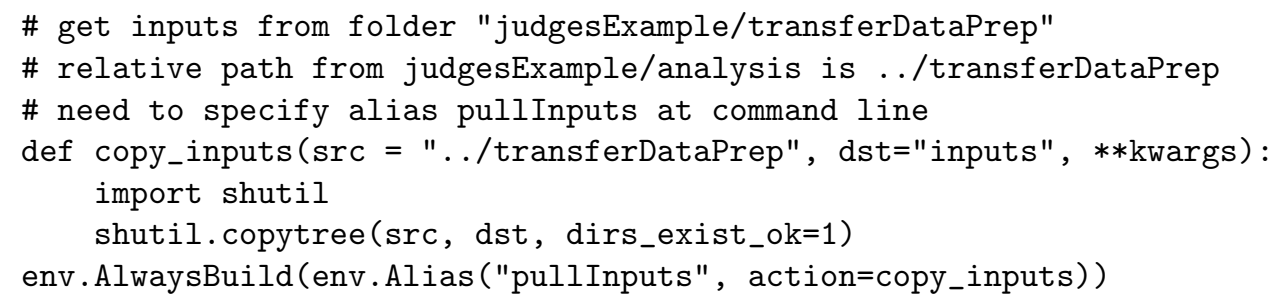

While we have made the transferDataprep directory local in this example, it could be a shared folder such as on Dropbox or Google Drive. Each user would just have to

34. The dirs_exist_ok option of shutil.copytree requires Python 3.8 or higher. 
specify the right path in their config_local.ini configuration file. See Appendix B on collaborative workflows for additional comments.

\section{Python Commands - Download and Unzip Archives}

It is straightforward to use Python utilities in SCons. As an example, the data for our Applied Example are stored on Dropbox in a zip file. In dataprep/SConstruct, we include instructions to download and unzip the .zip file using Python utilities.

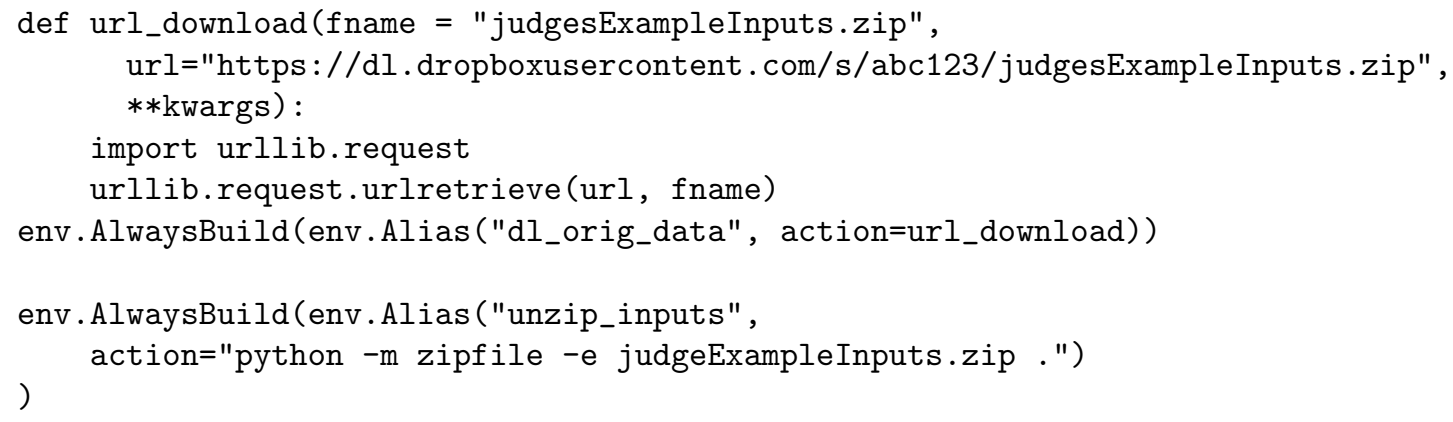

Since these actions do not have targets, their aliases must be specified in the call to statacons, e.g.,

statacons dl_original_data

statacons unzip_inputs

\section{Other Types of Extensions}

There are many other ways to add to SCons. We list a few resources here. First, there are several user-written Tools implemented as Python packages ${ }^{35}$ Second, the user can write Commands, Builders or Tools. See the SCons User Guide for documentation 36 A nice step-by-step example is provided by Wichman (2021).

\subsection{Alternative Approaches}

\section{Alternative Build Tools}

We chose to work in SCons for two main reasons. First, because it is a Python tool we could integrate it into Stata without requiring the use of a shell. Second, it provides the fundamental desirable features of build systems, as articulated in Mokhov et al. (2018): it is "minimal", in that it only rebuilds when dependencies have changed, and

35. See https://github.com/SCons/scons/wiki/ToolsIndex for a list.

36. As of Version 4.3.0 (December 2021), the relevant sections are 17, "Extending SCons: Writing Your Own Builder", and 18, "Not Writing a Builder: the Command Builder." 
only runs each task once, and it provides "early-cutoff optimization," knowing when a newly built object has not changed and then ceasing to rebuild downstream targets. There are many alternatives to SCons; in this section we list several and discuss what we see as the advantages and disadvantages relative to SCons.

The best-known build tool is make. SCons has a few advantages relative to make. First, make rebuilds targets when any dependency's timestamp is newer than the target, which leads to unnecessary rebuilds if a dependency's content has not changed 37 Second, make uses a custom syntax that is typically difficult for beginners (it uses many special characters and is unforgiving of rules for which whitespace characters to use). Third, make requires the use of a Unix shell (or emulator like Terminal on Macintosh or Windows Subsystem for Linux) to be used interactively (using shell make in Stata will not show you the output). One advantage of make over Scons is that it is somewhat easier to code straightforward commands into a script without having to create a build environment. A second advantage is the makefile2graph utility, which produces graphs of dependency trees. We are not aware of a similar utility for SCons.

There are many other build tools, such as cmake, some of which have support for using content signatures instead of file timestamps, but most are not in Python and so can not be used interactively inside of Stata. Among Python-based build tools, we chose to work in scons because its widespread use means there are active discussion and support communities.

The user-written project command in Stata (Picard 2013) is an ingenious pure Stata approach: dependencies are encoded into a project's do-files and the project program maintains a database of signatures to decide which outputs to rebuild. We prefer working in SCons for a few reasons, including integration with other tools, configurability, and access to advanced option such as the cache and parallel jobs, and that it does not require making changes to existing do-files.

\section{Literate Programming}

A more popular approach to reproducible research in Stata has followed the literate programming paradigm, in which text and code are combined in a single document. There are several excellent options in this paradigm, including built-in Stata commands such as dyndoc and putdocx, "notebooks" like the Jupyter notebooks popular in Python and now accessible in Stata 17, and user-written commands like texdoc (Jann 2016), webdoc (Jann 2017), markdoc (Haghish 2016), and markstat (Rodríguez|2017).

The literate programming approach is appealing and is certainly useful in many cases ${ }^{38}$ A literate programming script does provide a degree of self-documentation since input and output file names are present in the same file as the text. However, literate programming has limitations similar to those of a a master do-file: as projects grow more complex and computationally intensive, either all analyses must be repeated

37. SCons can replicate this behavior if desired, see the Decider() function mentioned in Section 3.3

38. For example, we used texdoc with statacons to produce Section 2 of this paper, dyndoc with statacons to produce the companion tutorial website, and markdoc to produce our help files. 
every time the document is produced, or the user must make some manual decisions about which parts to refresh and which to skip. See our discussion of separation of concerns in Section 4. Fortunately, a build tool such as statacons can complement a literate programming tool by automating some of these decisions. For example, our web tutorial consists of several web pages, each produced by a corresponding dyndoc file. We have written an SConstruct to manage this process, so that we rebuild a web page if and only if the inputs have changed. This code is available on our Wiki page 39

\subsection{Limitations}

The main limitation we have found in working with SCons is that SCons only recognizes builds it has performed itself. That is, SCons determines which targets need to be rebuilt by comparing the current state of the project to its state after the last time a project was built by SCons, as recorded in the sconsign.dblite database 40

As an example, suppose that in our Introductory Example, the user has edited analysis.do to change an axis title in the target scatterplot.pdf. The user runs her code from the Stata command window in the usual way (do code/analysis.do) and is satisfied with the result. In reality, the targets scatterplot.pdf and regressionTable.tex are now up to date. However, SCons will not realize this - SCons will compute a signature of analysis.do, see that it has changed since its signature was last recorded in sconsign.dblite, and believe that the targets need to be rebuilt.

In make, if the user knows that a build is up-to-date, the --touch option will change targets' timestamps to the present. Since make rebuilds only when dependencies are newer than the target, make will no longer rebuild the target.

SCons does not have a similar option, so we have added the assume_built and assume_done options to the statacons command, in which the user can designate certain targets that do not need to be rebuilt or do-files that do not need to be re-run. If the already built targets all have newer timestamps, then our provided "contenttimestamp-newer" Decider () can also be used. See the respective entries in Section 3.2

A second, related limitation is that setting up a collaborative project, for example keeping code under version control (e.g., Git) and sharing files through a service like Dropbox, requires a bit of care. Our preferred approach is a shared SCons cache. This is a powerful tool provided by SCons but there is a learning curve. We describe this method and some alternatives in Appendix B.

Finally, as mentioned in Section 6.2, it is easier to implement one-off tasks with make than with SCons ${ }^{41}$ We try to reduce this limitation by providing a few examples that users can adapt, but some familiarity with Python coding and some trial-and-error will likely be required to extend these examples to other tasks.

39. https://github.com/bquistorff/statacons/wiki/Statacons-and-literate-programming. 40. This applies for all settings of the Decider() function, including timestamp-newer. 41. At least, it is easier if the user is already comfortable using a shell and shell commands. 


\section{Conclusion}

In this paper, we have provided introductions to build tools and statacons, a build tool for Stata we have adapted from SCons. statacons runs directly in Stata without an external shell, can be used with a minimum of initial configuration, and does not require modification of existing code. While there is a startup cost to integrating statacons into a project, and it requires some discipline to keep SConstructs up to date, the benefits - reliable reproducibility and saved computation time - are considerable.

We recommend that users start small: while retrofitting a large, existing project may prove frustrating for new users, using statacons and a build-tool mindset from the beginning of a new project can promote better code by encouraging breaking down tasks into parts, simplifying the scope of individual pieces of code, and separation of concerns. In debugging problems, starting small is also advised - first, run code in Stata to see if the problem is with the code rather than the build tool, then build one or a few targets at a time until the problem is isolated, examine the information provided by the debug, tree and show_config options and the stataconsign function 42

It is our hope that this paper will encourage the use of build tools in Stata. One barrier is that there are few worked examples that are simple enough for the novice to understand easily but also show some of the power of the tool. We provide a few such examples in this paper and the accompanying material. We hope that other users will build on this work by providing additional annotated examples and by extending the scope of tasks that can be performed. We encourage users to share these advances publicly, and we provide a project Wiki to host such contributions.

\section{Acknowledgements}

We thank Riley Wilson for generously agreeing to our request to use Shumway and Wilson (2021) as the applied example in this paper. We thank the editor, Prof. Stephen P. Jenkins, and an anonymous referee for comments that improved the paper.

\section{References}

BITSS. 2020. Guide for Advancing Computation Reproducibility in the Social Sciences. Berkeley Initiative for Transparency in the Social Sciences. https://bitss.github.io/ACRE.

Christensen, G., Z. Wang, E. L. Paluck, N. Swanson, D. J. Birke, E. Miguel, and R. Littman. 2019. Open Science Practices are on the Rise: The State of Social Science (3S) Survey. preprint, MetaArXiv. https://osf.io/5rksu.

Gentzkow, M., and J. M. Shapiro. 2014. Code and Data for the Social Sciences: A Practitioner's Guide. University of Chicago mimeo. http://web.stanford.edu/gentzkow/research/CodeAndData.pdf.

42. The do-file debugging-checklist.do included with the statacons package may be useful. 
Haghish, E. F. 2016. Markdoc: Literate Programming in Stata. The Stata Journal 16(4): 964-988. https://doi.org/10.1177/1536867X1601600409.

Jackson, M. 2016. Software Carpentry: Automation and Make. http://swcarpentry.github.io/make-novice/.

Jann, B. 2005. ESTWRITE: Stata module to store estimation results on disk. Statistical Software Components, Boston College Department of Economics. https://github.com/benjann/estwrite.

— 2007. Making Regression Tables Simplified. The Stata Journal 7(2): 227-244. https://doi.org/10.1177/1536867X0700700207.

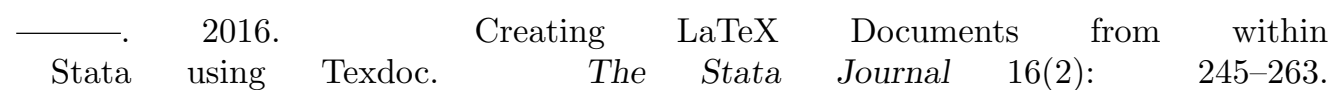
http://journals.sagepub.com/doi/10.1177/1536867X1601600201.

- 2017. Creating HTML or Markdown Documents from within Stata using Webdoc. The Stata Journal 17(1): 3-38. http://journals.sagepub.com/doi/10.1177/1536867X1701700102.

Mokhov, A., N. Mitchell, and S. Peyton Jones. 2018. Build systems à la carte. Proceedings of the ACM on Programming Languages 2(ICFP): 1-29. https://dl.acm.org/doi/10.1145/3236774.

Orozco, V., C. Bontemps, E. Maigné, V. Piguet, A. Hofstetter, A. Lacroix, F. Levert, and J.-M. Rousselle. 2020. How to Make a Pie: Reproducible Research for Empirical Economics and Econometrics. Journal of Economic Surveys 34(5): 1134-1169. http://onlinelibrary.wiley.com/doi/abs/10.1111/joes.12389.

Picard, R. 2013. PROJECT: Stata module providing a set of tools to build and manage a Stata project. Statistical Software Components, Boston College Department of Economics. https://ideas.repec.org/c/boc/bocode/s457685.html.

Rodríguez, G. 2017. Literate Data Analysis with Stata and Markdown. The Stata Journal 17(3): 600-618. http://journals.sagepub.com/doi/10.1177/1536867X1701700304.

SCons Development Team. 2021a. SCons 4.3.0 Man page. https://scons.org/doc/4.3.0/PDF/scons-man.pdf.

—. 2021b. SCons 4.3.0 User Guide. https://scons.org/doc/4.3.0/PDF/sconsuser.pdf.

Shumway, C., and R. Wilson. 2021. Workplace Disruptions, Judge Caseloads, and Judge Decisions: Evidence from SSA Judicial Corps Retirements. Working Paper, forthcoming, Journal of Public Economics. https://go.ncsu.edu/shumway.wilson.judges.

Stodden, V., J. Seiler, and Z. Ma. 2018. An empirical analysis of journal policy effectiveness for computational reproducibility. Proceedings of the National Academy of Sciences 115(11): 2584-2589. http://www.pnas.org/lookup/doi/10.1073/pnas.1708290115. 
Sun, L., and S. Abraham. 2021. Estimating dynamic treatment effects in event studies with heterogeneous treatment effects. Journal of Econometrics 225(2): 175-199. https://www.sciencedirect.com/science/article/pii/S030440762030378X.

Vega Yon, G. G., and B. Quistorff. 2019. parallel: A command for parallel computing. The Stata Journal 19(3): 667-684. https://doi.org/10.1177/1536867X19874242.

Wichman, M. 2021. ToolsForFools. https://github.com/SCons/scons/wiki/ToolsForFools.

Wilson, G., D. A. Aruliah, C. T. Brown, N. P. Chue Hong, M. Davis, R. T. Guy, S. H. D. Haddock, K. D. Huff, I. M. Mitchell, M. D. Plumbley, B. Waugh, E. P. White, and P. Wilson. 2014. Best Practices for Scientific Computing. PLoS Biol 12(1): e1001745. http://dx.doi.org/10.1371/journal.pbio.1001745.

\section{About the authors}

Guiteras is Assistant Professor in the Department of Agricultural and Resource Economics and Core Faculty in the Global WaSH Research Cluster at North Carolina State University.

Kim is a Ph.D. student in Economics at North Carolina State University.

Quistorff is a Research Economist in the Office of the Chief Economist at the Bureau of Economic Analysis.

Shumway is a Ph.D. student in Economics at North Carolina State University.

The authors are listed alphabetically. All views expressed in this paper are our own and do not necessarily reflect those of our employers or any other institutions with which we are affiliated.

Quistorff is the corresponding author, though for concerns regarding the package (additional information, questions, suggestions, and bug reporting) please use our project repository: https://github.com/bquistorff/statacons/

For the latest version of the software and installation instructions, go to the project web page: https://bquistorff.github.io/statacons

For previous versions of the software and installation instructions, go to our project archive https://osf.io/gbh4m/ 


\section{Installation Guide}

\section{Requirements}

These installation instructions correspond to statacons v3.0.0, released with this version of the paper. For up-to-date installation instructions, corresponding to the latest version of statacons, see our project web page: https://bquistorff.github.io/statacons

The following are required for statacons:

- Stata 16.0 or later

- Python 3.5 or later 43

- SCons 4.2 or later

\section{Step 1: Python Setup}

We provide the basic steps for installing Python and configuring Stata for use with Python. For more details, we recommend a series of posts by Chuck Huber on the Stata Blog ${ }^{44}$

Python commands are issued at a command prompt in a terminal window. Exactly which terminal will depend on your operating system and implementation of Python. Some popular examples include

- Windows

- Python: Windows Command Prompt ("cmd.exe") or Powershell

- Anaconda: Anaconda Powershell Prompt or Anaconda Prompt

- macOS: Terminal

- Unix-like (e.g., Linux): terminal

\section{Step 1.1: Install Python}

Install Python 3.5 or later (3.8 or later recommended).

\section{Step 1.2: Configure Stata to work with Python}

\section{Step 1.2.1: Locate Python executable}

In Stata, enter

43. Some of the advanced options in our Applied Example require Python 3.8 or later.

44. https://blog.stata.com/tag/python/ see especially posts 1 Huber, C., "Stata/Python integration part 1: Setting up Stata to use Python" and 3 Huber, C., "Stata/Python integration part 3: How to install Python packages"). 
python search

to locate the Python executable. If your system has both Python 2 and Python 3 installed, be sure to use the Python 3 executable.

\section{Step 1.2.2: Set path to Python executable in Stata}

Stata will use the default Python, unless configured before first use. To do so, in Stata, enter

python set exec pyexecutable, permanently

where pyexecutable is a path found in Step 1.2.1.

If Stata is started in a specific Anaconda environment, we provide a simple command in Stata to query the environment and set the Python path accordingly.

set_python_exec_env

\section{Step 1.2.3: check setup}

In Stata, check your setup by entering

python query

\section{Step 1.3: Install Python packages scons and pystatacons}

pip is the standard way to install Python packages. In the terminal, enter

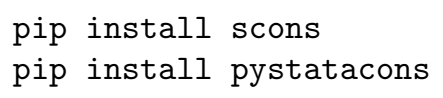

To check that these are installed and recognized by Stata, open Stata and enter

python which SCons // case-sensitive

python which pystatacons

\section{Step 2: statacons installation and setup}

\section{Step 2.1: Install statacons Stata package}

First, check your adopath. By default, statacons will be installed to your PLUS directory. Users working in collaboration with others may wish to change the PLUS and PERSONAL directories to be local to the project so that all users will be using the same version of user-written do-files. See the profile_template.do we have included in our project files for what we recommend. 
Second, statacons requires both the core program files and a key set of project files. You may wish to install these separately. To install the main program use:

net install statacons, from(https://raw.github.com/bquistorff/statacons/main/).

The core program files are:

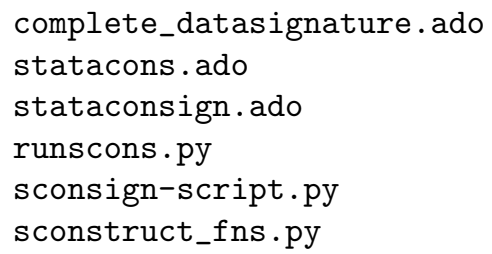

To check that statacons is installed, enter which statacons in Stata.

\section{Step 2.2: Install statacons project files}

To install the project files, navigate to the root of your project directory and then run

// Change to project's root directory cd path/to/your/project

// set other to the current directory, i.e. project's root directory net set other.

// get project files

net get statacons, from(https://raw.github.com/bquistorff/statacons/main/)

unzipfile project_files

rm project_files.zip

The project files packages in project_files.zip are

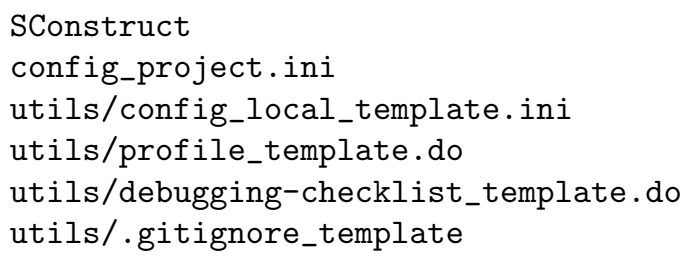

The only project file required is SConstruct. You may also wish to use the template files; if so, copy them into the main project folder (removing the _template suffix).

\section{Step 2.3: Configuration}

statacons is intended to work without additional user configuration, for example by automatically detecting the location of the Stata executable. However, some configura- 
tion may be required. For example, if you have more than one version of Stata installed, statacons may not find your preferred version first.

Our default is to use two configuration files, config_project.ini, which sets parameters that are common to all users working on a given project, and config_local.ini, which sets parameters specific to the local user. The files config-project.ini and config_local_template.ini provided with the Stata project files give several examples of how to set commonly-used parameters. See Section 3.4 for more information on these configuration files and how to read from them in SConstructs.

The default is that these configuration files, if used, will be stored in the same directory as the SConstruct. The command line option config_file() can instruct SCons to use a custom location or filename instead.

Users can specify other locations and names for configuration files using the config_file() option, see Sections 3.2 and 3.4

To check the configuration of statacons, enter statacons, show_config in Stata. The do-file debugging-checklist.do included in the utils folder may also be useful in checking that components are in the right places.

\section{Step 2.4 (optional): Setup for Version Control}

If you are using version control software such as git, here are a few recommendations:

- set your ado-path so that statacons and other packages are installed in the project folder. This will ensure that all users have the same version of all ado-files. See profile_template.do in the utils folder

- files to keep under version control: SConstruct, config_project.ini

- files to exclude from version control: config_local.ini, debugging-checklist.do, all generated files, including SConsign files (e.g., .sconsign.dblite) and batchgenerated .log-files. See .gitignore_template in the utils folder for what we recommend.

See Appendix B for more details on working with version control.

\section{Step 3: Test run with Introductory Example}

Replication code and data for our Introductory Example as well as the extensions to that example are provided in stataconsIntro.zip. posted to our repository 45 To run the example, unpack the zip archive and follow the steps in Section 2 of the paper.

45. https://github.com/bquistorff/statacons/main/raw/examples/stataconsIntro.zip 


\section{A Other Advanced Features}

\section{A.1 SConscripts and Hierarchical Builds}

SConscripts allow dividing a project into parts. Figure A1 shows how we can divide our Introductory Example into separate SConscripts for dataprep and analysis. Note the Export() and Import() statements needed to pass environments and variables across the SConstruct and the SConscripts.

In addition to breaking up large SConstructs into more manageable, readable parts, using SConscripts allows assigning different options or parameters to different parts of the project.

\section{A.2 Parallel Builds}

SCons can manage parallel builds, conducting tasks that are not interdependent simultaneously by dividing the computer's processors among the tasks. This can reduce computation time relative to running tasks sequentially.

At present, parallel builds are only supported in SCons itself, i.e., running scons from a terminal, not in statacons.

Using parallel builds without any modification to code can reduce computation time. Furthermore, writing code with parallel builds in mind can unlock additional gains. In our Applied Example, the event study methodology of Sun and Abraham (2021) is conducted for four different variables. Each estimation is independent of the others, so the four can be conducted in parallel. Figure A2 provides the SConscript for this process. We use an SConscript so that we can set the number of separate jobs for these tasks without affecting the rest of the build. Note the SetOption('num_jobs' ,2) line in the SConscript. We use 2 as a minimal working example, users with more processors may wish to allow more jobs.

We have included the do-files for this parallel build in our Supplemental Materials online. The basic idea is that (1) for each outcome variable, we (a) create a dataset with that variable and the relevant controls and identifiers, (b) perform the estimate for that outcome and save the results in a dataset, then (2) once the estimation is complete for all four variables, we combine the estimation results for the four outcome variables and present the results.

The gains from parallel builds will depend on many factors, including the nature of the task, the hardware, version of Stata, file size, and the system I/O throughput. For example, User A with 8 processors but a 4-core version of Stata MP will likely experience greater relative gains by adding a second, parallel job than User B with 8 processors and 8-core Stata MP: User A was only using 4 processors previously, and now can use all 8 simultaneously, still applying 4 to each task; User B was previously devoting 8 processors to each task sequentially, and now will devote 4 processors to each 
task simultaneously ${ }^{\mathrm{A} 1}$. Similarly, tasks that use only a small fraction of the available RAM can run in parallel without much cost to the speed of the individual tasks, while tasks that are demanding of available RAM may not be sped up as much when run in parallel. Finally, as noted in the paper, parallel (Vega Yon and Quistorff 2019) is a better option for bootstrapping or simulation.

Some care should be taken in writing code in these cases - while there is no issue with multiple Stata processes accessing the same dataset at once, it is important that parallel tasks not write to the same dataset. This just requires a bit of caution that no output filenames are repeated across jobs.

$A 1$. For some rough guidance on the types of tasks more or less likely to benefit from parallel builds, see the "Stata/MP Performance Report" (https://www.stata.com/statamp/report.pdf) for data on speed gains as the number of cores increases for different commands. Note that the gains as the number of cores increases is negatively related to gains from parallel builds if a fixed number of cores are being divided among tasks. 


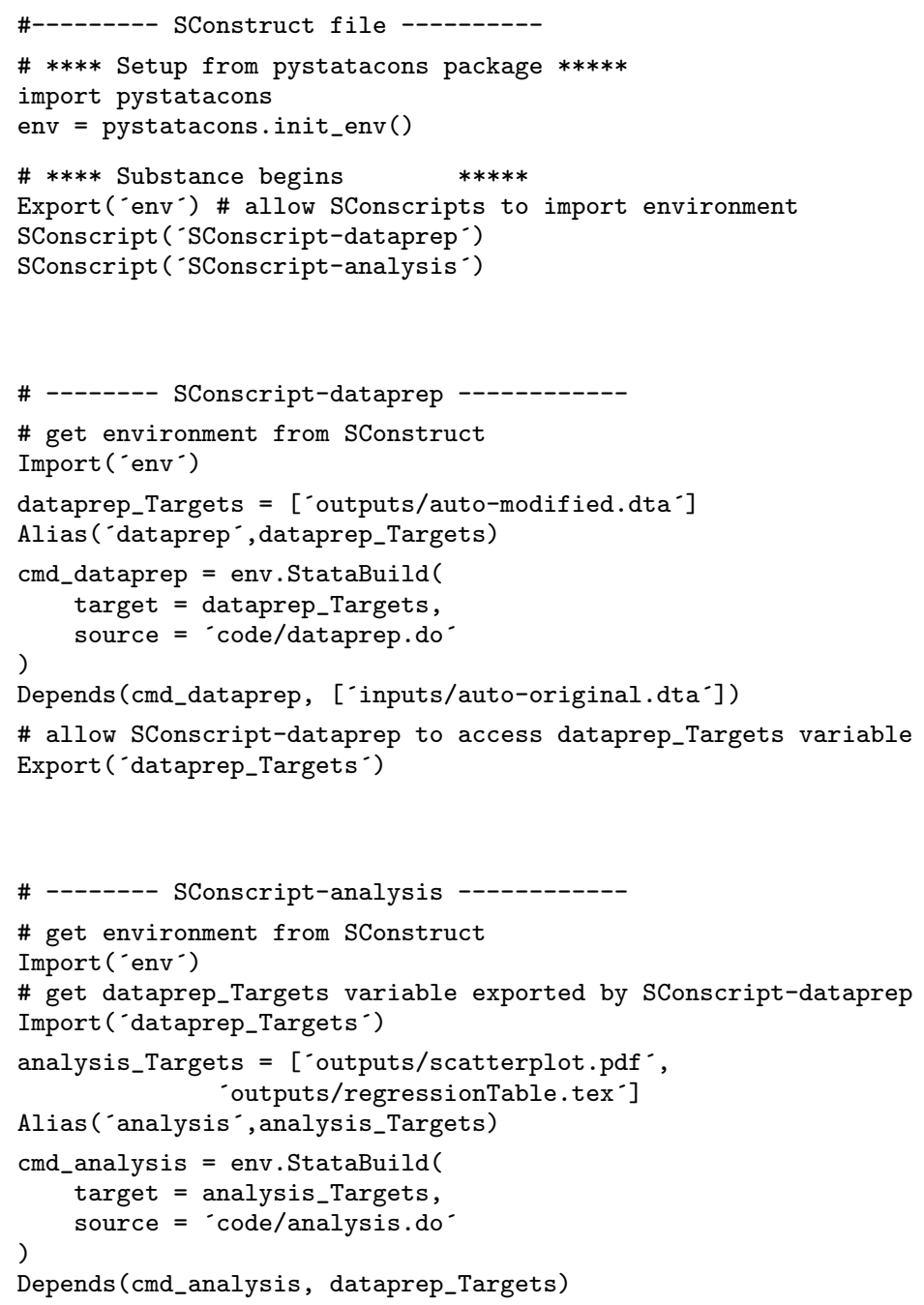

Figure A1: An SConstruct file with two SConscript files 


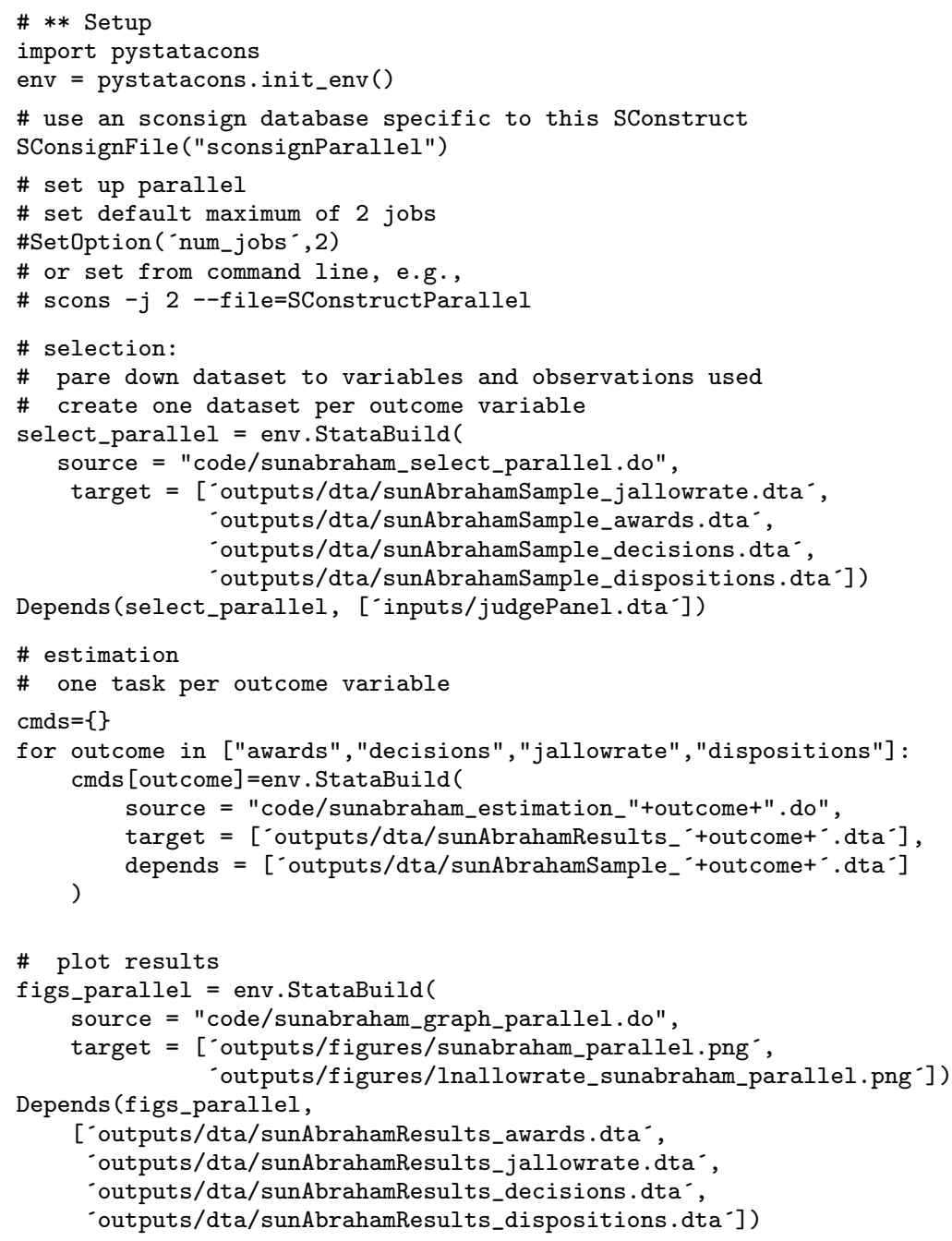

Figure A2: SConstruct: Parallel Jobs in Applied Example 


\section{B Collaborative Workflows}

\section{The Problem}

In this Appendix, we discuss a few models for collaborative workflows. The main difficulty with collaboration is that, as we mention in the Limitations section $(6.3)$, SCons only understands builds it has done itself. That is, SCons compares the current state of targets and dependencies to their state as recorded in the .sconsign.dblite database the last time SCons ran the build.

As an example, suppose that Users A and B are collaborating on the Introductory Example of Section 2. User A is working primarily on the dataprep task and User B is working primarily on analysis. Imagine for the sake of this example that the dataprep task takes a very long time to run. User A has made some edits to dataprep.do and updated auto-modified.dta. User A shares these two files with User B.

The issue is that User B's SCons will look at dataprep.do, compare its current status to its status the last time auto-modified.dta was built as recorded in User B's .sconsign.dblite, see that dataprep. do is different, and conclude that auto-modified.dta needs to be rebuilt. User B could use the assume_built() or assume_done() options we have added to SCons to tell SCons that in fact auto-modified.dta is up to date, but in more complex projects this sort of mental dependency-tracking is prone to failure.

The underlying problem is that there are three competing values: we want builds to be complete (all targets up-to-date), we do not want to duplicate work, and we want to automate the build and avoid manual workarounds, which are error-prone. Our preferred approach is to use the SCons cache to share built files. While this requires a small amount of effort to set up and some care to maintain, it satisfies all three values without introducing too much additional complexity. We will describe this approach below, then mention a few alternatives and their limitations. Our project Wiki contains a simple worked example of a collaborative project using the SCons cache along with GitHubB1

\section{SCons Cache}

Our preferred approach is to use the SCons cache to share derived files. See Figure B1 for an example SConstruct. In the SConstruct, we designate a shared folder to store the cache:

\section{CacheDir ('path/to/cache')}

Rather than hard-code the path into the SConstruct, the path can be coded into a configuration file (e.g., config_project.ini, config_local.ini, or other specified through the config_file() option), which the SConstruct can then read. Here, we use a local scons_cache folder so that this can be a self-contained example, but in practice this may be a shared drive or a folder on a file-sharing service. See config_local_template.ini

B1. https://github.com/bquistorff/statacons/wiki/Collaboration-using-GitHub-and-the-SCons-cache 
for several examples.

SCons will then store built files in the cache and, before building targets, check to see whether an up-to-date version is available in the cache. See the SCons User Guide for details on how this works B2

We have found that this approach handles the three competing goals of a build tool (complete builds; reduce unnecessary builds; automaticity instead of manual intervention) well, with a few caveats.

The main caveat is that by default SCons will update the cache whenever a target is newly built, which is not desirable while writing and testing code since it will lead to filling the cache with unnecessary files, and other users will be repeatedly downloading these unnecessary files. It is good practice to update the cache only when you are sure the work is complete. There are two ways to do this.

The first way is to use the command-line option cache_readonly to tell SCons to check and read from the cache but not update the cache with any built files, or cache_disable to tell SCons to ignore the cache entirely. Once you have successfully completed your build, use the option cache_force to force SCons to update the cache. This will also make it less likely that two users will attempt to update the same cached file at the same time.

The second way is to edit the SConstruct so that the default is to have the cache disabled, and a command-line option is required to read to or write to the cache. We provide an example on our project Wiki B3

A few additional caveats. First, the cache can get large and periodically need to be cleaned and rebuilt ${ }^{B 4}$ Second, when using the cache, SCons calculates build signatures regardless of the Decider method chosen, so there are no time savings from using, for example, content-timestamp. Of course, this does not affect those using the default content. Finally, in its own storage, the cache uses file signatures as filenames, instead of the cached file's own name, which is slightly inconvenient if you need to find and examine the cached version of a particular file. The cache_debug option will provide information on the files being used, and note that the file will be stored in a subfolder corresponding to the first two characters of the cached filename. For example, a cached file d7581eba1ac59ad5f92b2fceba04477f will be in subfolder D7.

We list some useful command-line options for the cache below. Using these options together with dry_run can be instructive to get a preview of how SCons will interact with the cache.

We list the Stata-style syntax first, then the standard SCons syntax. Recall that either syntax is allowed but the two cannot be mixed in a single command.

B2. As of SCons 4.3.0 (December 2021), this is Section 22, "Caching Built Files." See especially the first footnote in that section, which describes the information SCons stores and uses to determine whether a target is up to date.

B3. https://github.com/bquistorff/statacons/wiki/Cache-with-cache-disabled-as-default.

$B 4$. Rebuilding the cache does not require rebuilding the project, just erasing the cache and then calling SCons with the option cache_force. 
cache_debug (-) prints information on the cache files being used to the screen

SCons equivalent: --cache-debug=-

cache_debug ( $f i l e$ ) saves information on the cache files being used to the file file

SCons equivalent: -- cache-debug=file

cache_disable do not use files from the cache, do not write files to the cache

SCons equivalent: --cache-disable, --no-cache

cache_force write all derived files to the cache, whether they are built in this call to

SCons or have previously been built. This overrides the default, which is to write only files that are built or rebuilt files in the current call to SCons.

SCons equivalent: --cache-force, --cache-populate

cache_readonly use files from the cache but do not write files to the cache

SCons equivalent: --cache-readonly

cache_show by default, SCons will print "retrieved file from cache" to the screen when it pulls a file from the cache rather than building it. With the cache_show option, SCons will instead print what it would have done to build the file if it had not used the cache.

SCons equivalent: --cache-show

\section{Other Options}

Here are a few alternatives, along with what we see as their main drawbacks.

\section{Shared .sconsign.dblite database}

Since SCons uses the .sconsign.dblite database to decide which targets need to be rebuilt, it is tempting to use a shared database, whether through a file-synchronization service like Dropbox or version-control system like Git or GitHub. However, this is unlikely to work well. Since the .sconsign.dblite is a single, binary file, version conflicts or file corruption can arise even if users are working on different aspects of a project. Furthermore, unless all users have all their code, targets and dependencies synchronized at all times, a shared .sconsign.dblite database will cause SCons to get very confused, since it will see discrepancies between files and database entries every time any user updates any file. This might be approximately workable if all users are synced to the same shared folder, e.g., a shared Dropbox folder, but this is not possible if one is using more full-featured source code and collaboration management solutions such as git. 


\section{Marking files as built}

The assume_built/assume_done options we have added for statacons can help in one-off cases. See 3.2 for their description. In the example above, User B could update to User A's latest dataprep.do and auto-modified.dta, then run

statacons, assume_built('outputs/auto-modified.dta')

SCons will then skip rebuilding auto-modified.dta, record the current file signature as in .sconsign.dblite, and move on to rebuilding the final outputs.

While this approach can be useful, it relies on users accurately keeping track of what is up to date and what is not, and making manual choices in builds, both of which build tools are intended to make unnecessary. For example, we have found assume_built (*) to be useful if a minor change to the builder has caused SCons to think all files need to be re-built when in fact none of them do. Again, though, we have to be sure that the change to the builder will not result in any substantive change.

\section{Additional Comments on Collaborative Workflows}

Version Control If you are using a version control system like Git or SVN, we recommend that you do not keep config_local.ini or .sconsign.dblite under version control. In the case of config_local.ini, this is simply because the purpose of this file is to account for things that will vary across users. In the case of .sconsign.dblite, see our discussion under 'Shared .sconsign.dblite database' above. There are a few additional Python-generated files (e.g., _-_pycache _- $_{\text {) }}$ that should not be kept under version control. In Git, this is handled with a .gitignore file. In the replication archive we provide for our Introductory Example, we have included an example .gitignore that excludes the files above. In addition, we recommend excluding all generated files. That is, as a rule, we keep only code (in the broad sense of things a human has typed, as opposed to things generated by the computer) under version control. This is generally considered good practice but is essential when using the SCons cache, since the cache is already essentially handling version control for generated files, and having both SCons and the version control software attempting the same task will lead to conflicts.

We do recommend keeping user-written programs under version control to ensure that all users have the same version of these programs. In our Introductory Example, we have kept user-written estout (Jann 2007) in the folder code/ado/plus. Our profile.do sets Stata's adopath to instruct Stata to look in this folder (and subfolders). Similarly, we keep config_project.ini, the SConstruct, statacons . ado and the other associated files under version control, again so that the build will be consistent across users. The downside of this practice is that you will need to maintain separate copies of the same programs for each project. 
Shared SCons cache If you are using Dropbox or a similar service to share your SCons cache, make sure that you have offline access to the folder containing the cache ${ }^{B 5}$ Using the "Smart Sync" or "Streaming Access" options that store files online and then fetch them when requested is likely to cause several problems. First, Python may not find folders that are only available in the cloud. Second, this will slow down the process of retrieving cached files. Third, you may not be able to access certain recently cached files when working offline.

Finally, as of April 2022 we do not recommend Google Drive for sharing caches. Google Drive will sometimes be able to discern the type of cached files and will add a file extension to the filename in the cache. This happens especially frequently with . pdfs. This will confuse SCons and prevent it from successfully retrieving that file from the cache. We have not found this to occur with Dropbox.

B5. As of April 2022: in Dropbox, either turn off "Smart Sync" to make all of your Dropbox content available offline, or use "Selective Sync" to keep your cache folders available offline. While we currently do not recommend Google Drive for shared caches (see the next paragraph), if you do use Google Drive, choose either "Mirror files" to make all of your Google Drive content available offline, or follow the instructions under "Stream files - Choose specific files and folders to make available offline" to keep your cache folders available offline. 


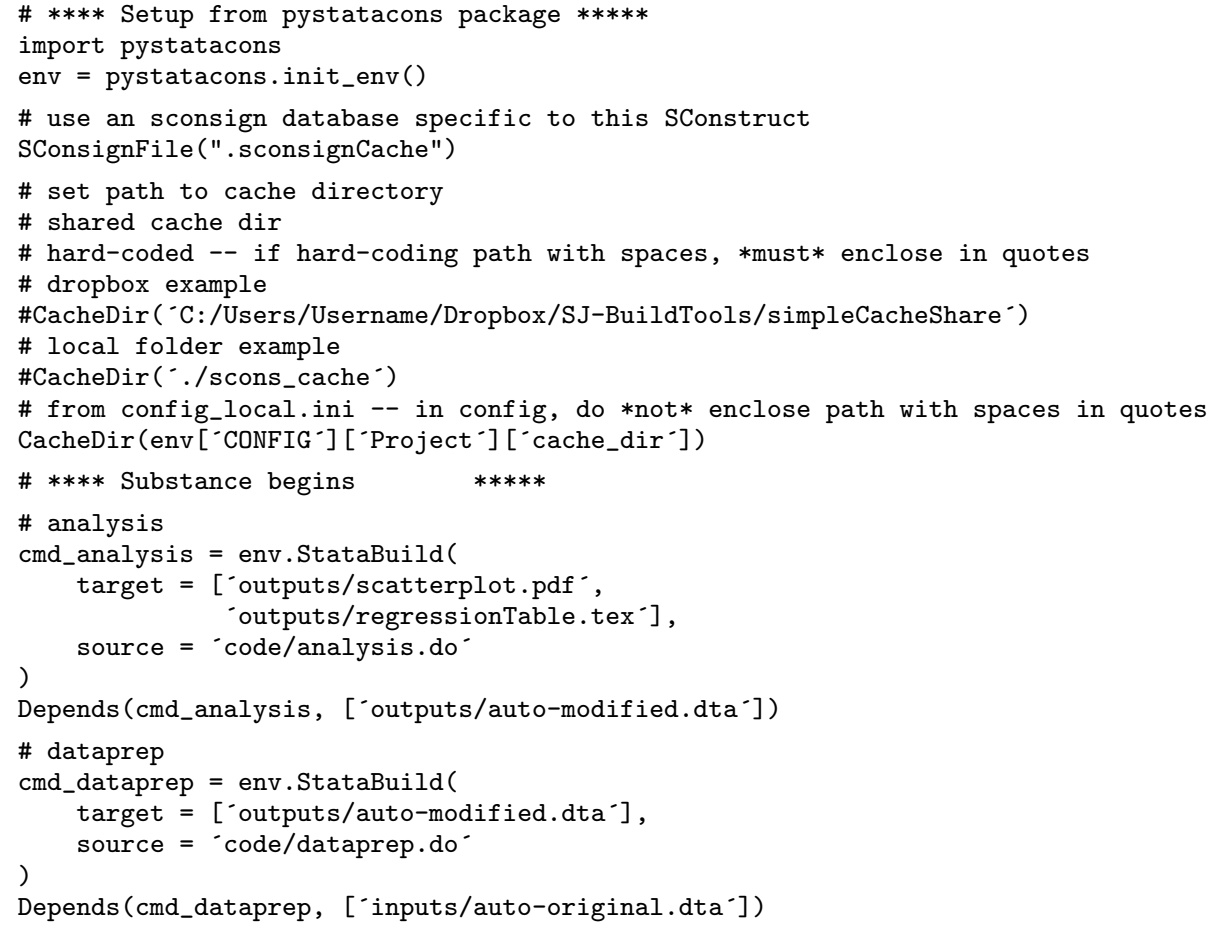

Figure B1: SConstruct for Introductory Example Using SCons Cache 
R. Guiteras, A. Kim, B. Quistorff, C. Shumway

\section{Compiling a PDF from the Introductory Example}

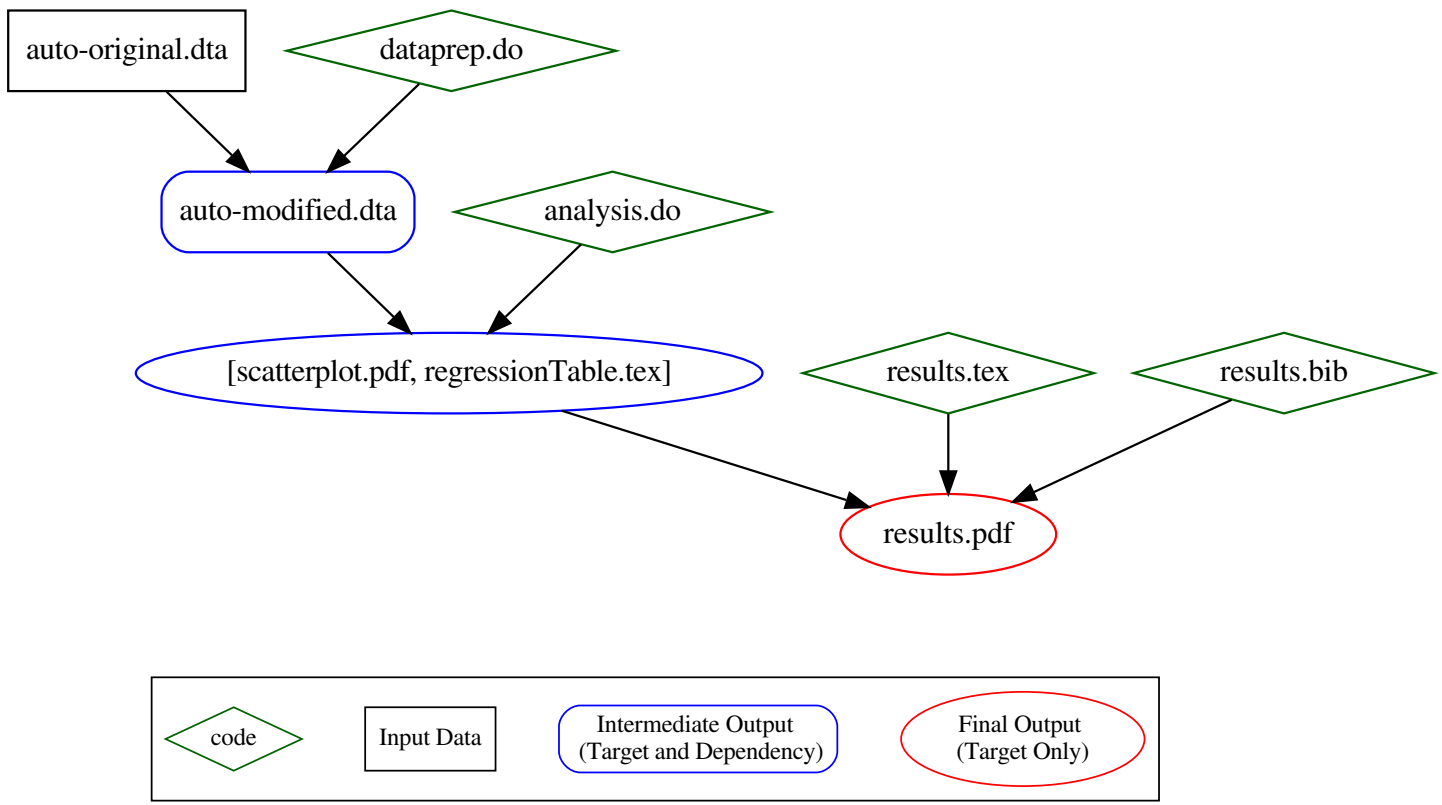

Figure C1: Workflow for Compiling PDF from Introductory Example 
\# SConstructCompilePDF

\# **** Setup from pystatacons package *****

import pystatacons

env = pystatacons .init_env ()

$\mathrm{Help}$ (" " "

To compile analysis targets only:

'statacons, file(SConstructCompilePDF)'

'scons --file=SConstructCompilePDF'

To clean only analysis targets (not compiled PDF)

'statacons, file(SConstructCompilePDF) clean'

'scons --file=SConstructCompilePDF -c'

To compile PDF:

'statacons compilePDF, file(SConstructCompilePDF)'

'scons --file=SConstructCompilePDF compilePDF'

To clean all targets plus all latex helper files (aux, bbl, etc)

'statacons compilePDF, file(SConstructCompilePDF) clean

'scons --file=SConstructCompilePDF compilePDF -c'

To clean only latex helper files (aux, bbl, etc)

'statacons cleanPDFhelp, file(SConstructCompilePDF) clean'

'scons --file=SConstructCompilePDF cleanPDFhelp -c'

" " ", append=True)

\# **** Substance begins $\quad * * * * *$

\# dataprep

dataprep_Targets $=\left[{ }^{\text {- outputs/auto-modified.dta }}{ }^{-}\right]$

Alias ('dataprep", dataprep_Targets)

cmd_dataprep = env.StataBuild (

target $=$ dataprep_Targets,

source $={ }^{\prime}$ code/dataprep.do',

depends $=\left[\right.$ 'inputs/auto-original.dta $\left.{ }^{-}\right]$)

\# analysis

analysis_Targets $=\left[\right.$ 'outputs/scatterplot.pdf $^{-}$, 'outputs/regressionTable.tex ']

Alias ('analysis', analysis_Targets)

cmd_analysis = env.StataBuild (

target $=$ analysis_Targets,

source = 'code/analysis.do",

depends = dataprep_Targets)

Default ( $a n a l y s i s^{-}$)

\# compile PDF - SCons knows how to scan tex files for dependencies env.Tool ("pdftex")

env. AppendUnique (PDFLATEXFLAGS $={ }^{-}$-quiet ${ }^{-}{ }^{\prime}$ )

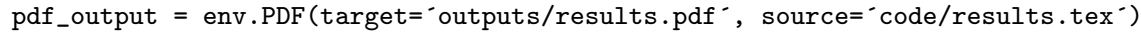

Alias ('compilePDF', 'outputs/results.pdf ")

\# extra files generated by pdflatex to clean

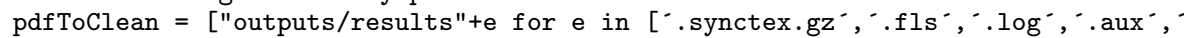

$>$.bbl ${ }^{-},,^{-}$.out $\left.\left.{ }^{-},{ }^{-} . \mathrm{dvi}^{-},,^{-} . \mathrm{blg}^{-}\right]\right]$

Clean ('cleanPDFhelp', pdfToClean)

Figure C2: SConstruct for Compiling PDF from Introductory Example 


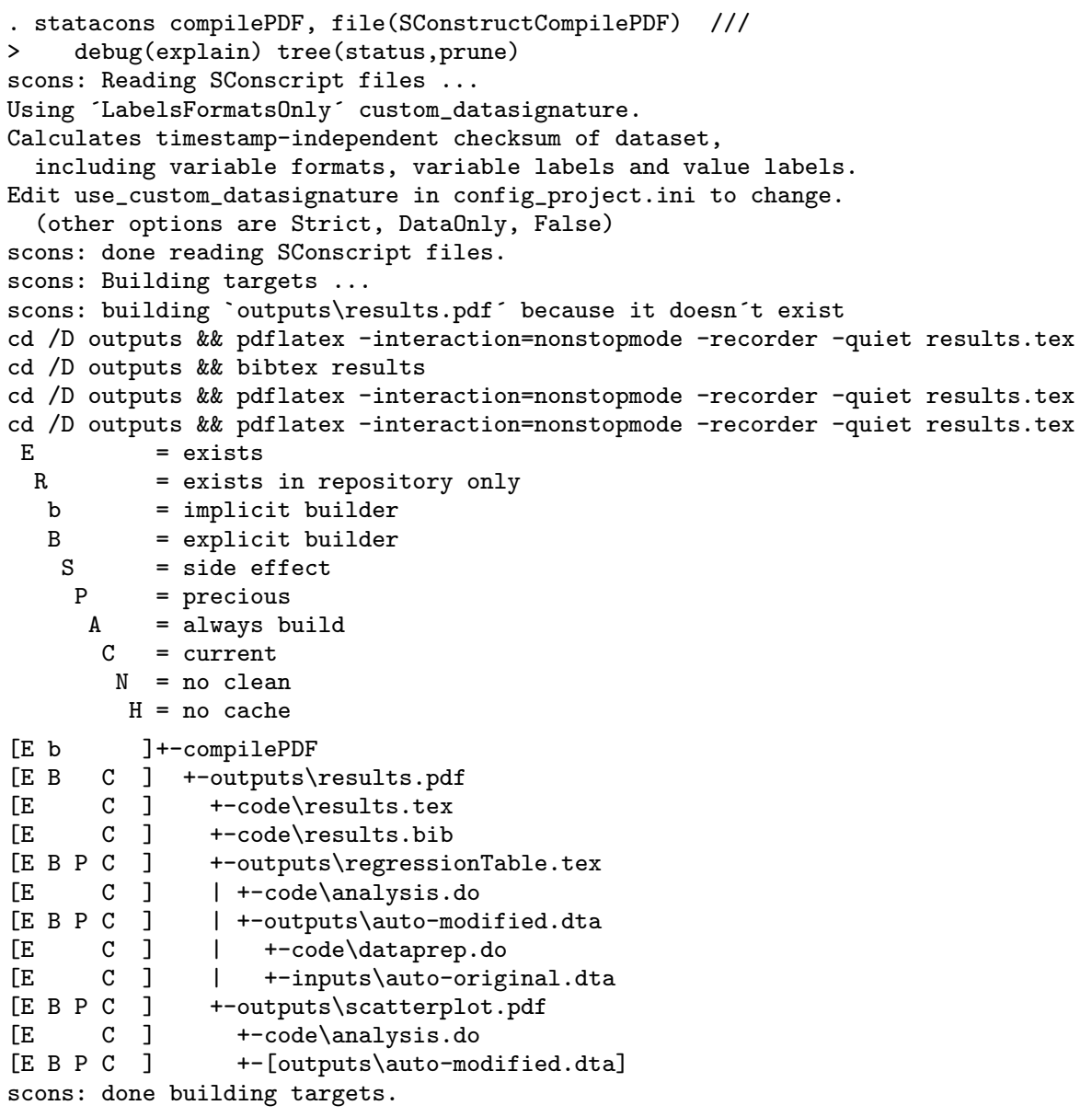

Figure C3: SCons output from Compiling PDF from Introductory Example 
statacons
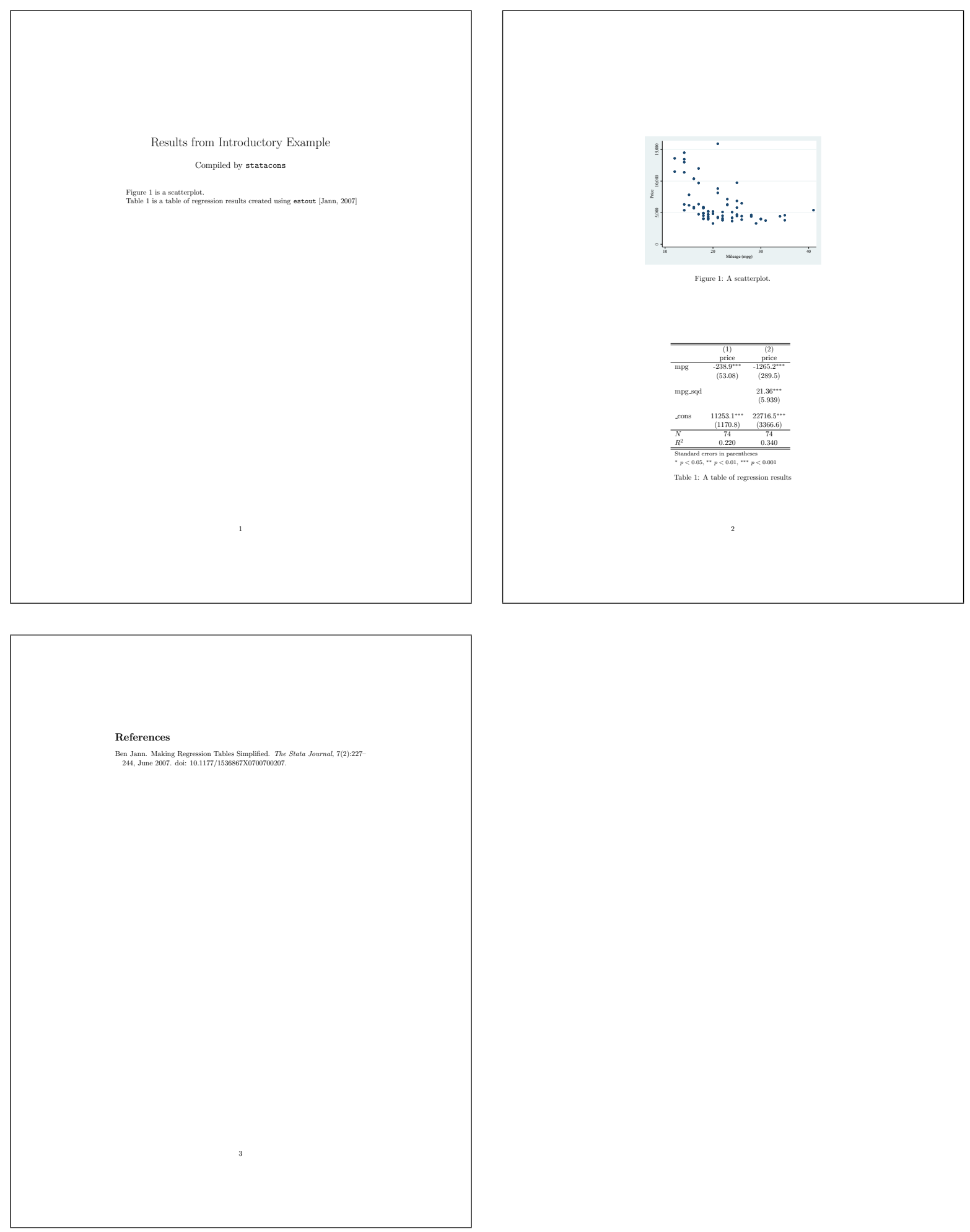

Figure C4: Compiled PDF from Introductory Example 


\section{Version History}

Most recent version of statacons: project repository https://github.com/bquistorff/statacons.

Most recent version of the paper: https://osf.io/preprints/metaarxiv/qesx6/.

Previous versions of statacons: https://osf.io/gbh4m/, see "Components" section .

Previous versions of the paper: https://osf.io/preprints/metaarxiv/qesx6/, click the "Download previous versions" button below the paper image, select desired version of the paper.

v3.0.0 May 2022 (this version, as resubmitted to The Stata Journal)

Citation: Guiteras, Raymond, Ahnjeong Kim, Brian Quistorff and Clayson Shumway, "statacons: An SCons-based build tool for Stata," CEnREP Working Paper 22001, May 2022, https://go.ncsu.edu/cenrep-wp-22-001. Under review at the Stata Journal.

Paper archive: https://doi.org/10.31222/osf.io/qesx6

Install statacons: net install statacons, from(https://raw.github.com/bquistorff/statacons/v3.0.0/)

Installation instructions: https://github.com/bquistorff/statacons/tree/v3.0.0

Code archive: https://osf.io/k4tqc/

v2.0.0 January 19, 2022

Citation: Guiteras, Raymond, Ahnjeong Kim, Brian Quistorff and Clayson Shumway, "statacons: An SCons-based build tool for Stata," CEnREP Working Paper 22001, January 2022, https://go.ncsu.edu/cenrep-wp-22-001. Under review at the Stata Journal.

Paper archive (v2): https://doi.org/10.31222/osf.io/qesx6, click the "Download previous versions" button below the paper image, select "Version 2."

Install statacons: net install statacons, from(https://raw.github.com/bquistorff/statacons/v2.0.0/)

Installation instructions: https://github.com/bquistorff/statacons/tree/v2.0.0

Code archive: https://osf.io/n42mc/

v1.0.0 January 4, 2022 (as submitted to The Stata Journal)

Citation: Guiteras, Raymond, Ahnjeong Kim, Brian Quistorff and Clayson Shumway, "statacons: An SCons-based build tool for Stata," CEnREP Working Paper 22001, January 2022, https://go.ncsu.edu/cenrep-wp-22-001. Under review at the Stata Journal.

Paper archive (v1): https://doi.org/10.31222/osf.io/qesx6, click the "Download previous versions" button below the paper image, select "Version 1."

Install statacons: net install statacons, from(https://raw.github.com/bquistorff/statacons/v1.0.0/) 
Installation instructions: https://github.com/bquistorff/statacons/tree/v1.0.0 Code archive: https://osf.io/cmh56/ 